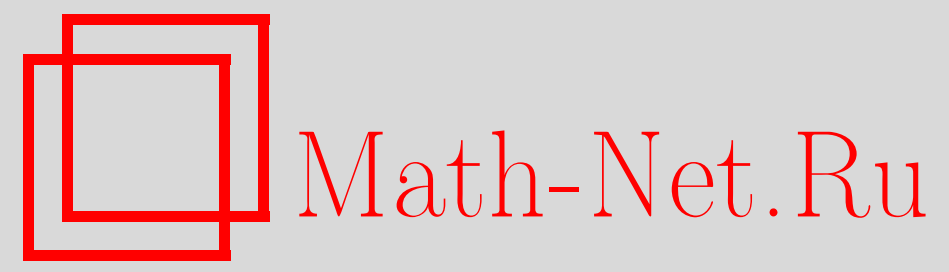

В. Н. Коновалов, Влияние формы функций на порядки кусочно полиномиальной и рациональной аппроксимации, Матем. сб., 2005, том 196, номер 5, 3-30

DOI: https://doi.org/10.4213/sm1355

Использование Общероссийского математического портала Math-Net.Ru подразумевает, что вы прочитали и согласны с пользовательским соглашением http://www.mathnet.ru/rus/agreement

Параметры загрузки:

IP: 52.87 .193 .239

26 апреля 2023 г., 09:46:21 
УДК 517.5

\section{В.Н. Коновалов}

\section{Влияние формы функций на порядки кусочно полиномиальной и рациональной аппроксимации}

Пусть $\Delta_{+}^{s}-$ множество функций $x: I \rightarrow \mathbb{R}$ на конечном интервале $I$ таких, что разделенные разности $\left[x ; t_{0}, \ldots, t_{s}\right]$ порядка $s \in \mathbb{N}$ этих функций являются неотрицательньми для всех наборов из $s+1$ различных точек $t_{0}, \ldots, t_{s} \in I$. Пусть $\Sigma_{r, n}=\left\{\sigma_{r, n}\right\}$ - множество кусочно полиномиальных сплайнов $\sigma_{r, n}$ порядка $r$ с $n-1$ свободными узлами, а $R_{n}=\left\{\rho_{n}\right\}$ - множество рациональных функций $\rho_{n}=\widehat{\pi}_{n} / \breve{\pi}_{n}$, где $\widehat{\pi}_{n}$ и $\breve{\pi}_{n}-$ многочлены порядка $n$. Для классов $\Delta_{+}^{s} B_{p}:=\Delta_{+}^{s} \cap B_{p}$, где $B_{p}$ - единичный шар в $L_{p}$, установлены точные порядки

$$
E\left(\Delta_{+}^{s} B_{p}, \Sigma_{r, n}\right)_{L_{q}} \asymp n^{-\min \{r, s\}} \text { и } E\left(\Delta_{+}^{s} B_{p}, R_{n}\right)_{L_{q}} \asymp n^{-s}
$$

наилучших приближений в метриках $L_{q}$ при условии, что $1 \leqslant q<p \leqslant \infty$.

Библиография: 18 названий.

\section{§ 1. Введение. Формулировки основных результатов}

При $s \in \mathbb{N}$ функцию $x: I \rightarrow \mathbb{R}$ будем называть $s$-монотонной на промежутке $I \subseteq \mathbb{R}$ (интервале, полуинтервале или отрезке), если для всех наборов из $s+1$ различных точек $t_{0}, \ldots, t_{s} \in I$ соответствующие разделенные разности $\left[x ; t_{0}, \ldots, t_{s}\right]$ порядка $s$ этой функции являются неотрицательными. Очевидно, что $s$-монотонные функции при $s=1$ и $s=2$ - это соответственно неубывающие и выпуклые функции на $I$. Таким образом, параметр $s$ характеризует форму функций.

Известны (см., например, [1]-[3]) следующие свойства $s$-монотонных функций. Если функция $x$ имеет непрерывную на $I$ производную $x^{(s)}$ порядка $s$, то эта функция является $s$-монотонной тогда и только тогда, когда $x^{(s)}(t) \geqslant 0, t \in I$. Ясно также, что у 1-монотонной функции $x$ сушествуют в каждой внутренней точке $t \in I$ конечные пределы $x_{-}(t)$ и $x_{+}(t)$ слева и справа. Если же $s>1$ и функция $x$ является $s$-монотонной на $I$, то при всех $k \leqslant s-2$ сушествуют обычные производные $x^{(k)}$ порядка $k$ в каждой внутренней точке $t \in I$, являющиеся $(s-k)$-монотонньми функциями на любом интервале из $I$. В частности, производная $x^{(s-2)}$ сушествует и является выпуклой функцией на любом интервале из $I$. Кроме того, $x^{(s-2)}$ удовлетворяет (локально) условию Липшица на каждом внутреннем отрезке из $I$. Следовательно, функция $x^{(s-2)}$ является локально абсолютно непрерывной на $I$. Односторонние производные $x_{-}^{(s-1)}(t)$ и $x_{+}^{(s-1)}(t)$ слева и справа сушествуют в каждой внутренней точке $t \in I$ и являются неубывающими функциями. Обычная производная $x^{(s-1)}$ сушествует почти всюду на $I$, за исключением не более чем счетного множества точек. 
Класс всех $s$-монотонных на $I$ функций будем обозначать через $\Delta_{+}^{s}(I)$. Кроме того, если на $I$ определен некоторый класс функций $W(I)$, то полагаем $\Delta_{+}^{s} W(I):=$ $\Delta_{+}^{s}(I) \cap W(I)$. Через $L_{p}(I), 1 \leqslant p \leqslant \infty$, обозначаем, как обычно, линейное пространство всех измеримых по Лебегу функций $x: I \rightarrow \mathbb{R}$ c конечной нормой $\|x\|_{L_{p}(I)}$. При $p=\infty$ под $\|x\|_{L_{\infty}(I)}$ понимается ess sup- или sup-норма. Единичный шар пространства $L_{p}(I)$ обозначаем через $B_{p}(I)$. Для промежутка $I$ с концами в точках $a:=-1$ и $b:=1$ его обозначение будет иногда опускаться. Например, если $I=(-1,1)$, то $W:=W(I)$.

Нетрудно проверить, что $\Delta_{+}^{s} B_{p} \not \subset L_{q}$ при $1 \leqslant p<q \leqslant \infty$. Следует также отметить, что несмотря на наличие при $s>1$ у функций $x \in \Delta_{+}^{s} B_{p}$ определенных дифференциальных свойств, нельзя, вообше говоря, гарантировать при $1 \leqslant p<\infty$ ограниченность норм производных $x^{(k)}$ порядка $k \geqslant 1$ в каком-либо из пространств $L_{q}, 1 \leqslant q \leqslant \infty$. Лишь для функций $x \in \Delta_{+}^{s} B_{\infty}$, где $s>1$, можно утверждать, что $\left\|x^{\prime}\right\|_{L_{1}}<\infty$.

Обозначим через $P_{n}(I)$, где $n \in \mathbb{N}$, множество всех алгебраических многочленов $\pi_{n}$ порядка $\leqslant n$ (т.е. степени $\left.\leqslant n-1\right)$, определенных на промежутке $I$. Через $R_{n}(I)$ обозначаем множество всех рациональных функций на $I$, имеющих вид $\rho_{n}=\widehat{\pi}_{n} / \check{\pi}_{n}$, где $\widehat{\pi}_{n}, \check{\pi}_{n} \in P_{n}$. При этом предполагается, что $\check{\pi}_{n} \not \equiv 0$. В тех точках $t \in I$, где $\check{\pi}_{n}(t)=0$, функция $\rho_{n}$ может принимать произвольные конечные значения. Если на промежутке $I$ с концами в точках $a:=-1$ и $b:=1$ задан некоторый класс $W$ функций $x$ такой, что $W \subset L_{q}$, то через

$$
E\left(W, R_{n}\right)_{L_{q}}:=\sup _{x \in W} \inf _{\rho_{n} \in R_{n}}\left\|x-\rho_{n}\right\|_{L_{q}}
$$

обозначим величину его наилучшего приближения в $L_{q}$ множеством $R_{n}$.

А.П. Булановым [4] было установлено, что для множества $\Delta_{+}^{2} B_{\infty}$ всех выпуклых на интервале $I:=(-1,1)$ функций $x$, удовлетворяющих ограничению $\|x\|_{L_{\infty}} \leqslant 1$, справедливы двухсторонние оценки

$$
c_{1} n^{-1} \leqslant E\left(\Delta_{+}^{2} B_{\infty}, R_{n}\right)_{L_{\infty}} \leqslant c_{2} n^{-1} \log ^{2}(n+1), \quad n \geqslant 1
$$

где $c_{1}>0$ и $c_{2}>0$ - абсолютные постоянные.

Затем В. А. Попов и П.П. Петрушев [5] усилили оценку сверху, доказав, что

$$
E\left(\Delta_{+}^{2} B_{\infty}, R_{n}\right)_{L_{\infty}} \leqslant c n^{-1}, \quad n \geqslant 1,
$$

где $c>0$ - абсолютная постоянная. Таким образом, был найден точный порядок

$$
c_{1} n^{-1} \leqslant E\left(\Delta_{+}^{2} B_{\infty}, R_{n}\right)_{L_{\infty}} \leqslant c_{2} n^{-1}, \quad n \geqslant 1,
$$

наилучших равномерных приближений класса $\Delta_{+}^{2} B_{\infty}$ рациональньми функциями.

К.Г. Иванов [6] также установил, что точный порядок наилучшего приближения в метрике $L_{1}$ класса $\Delta_{+}^{2} B_{\infty}$ ломаными, имеющими не более чем $n$ звеньев, равен $n^{-2}$.

Эти результаты ясно указали на то, что форма функций может существенньм образом влиять на порядки их приближения. Отметим, что вопросы аппроксимации классов $s$-монотонных функций из пространств Соболева исследовались 
в [7]-[12]. В [13], [14] изучалось поведение формосохраняюших поперечников типа Колмогорова классов $\Delta_{+}^{s} B_{p}$.

В настоящей работе решается задача о точных порядках наилучших приближений классов $\Delta_{+}^{s} B_{p}$ при всех $s \in \mathbb{N}$ в метриках $L_{q}$ для $1 \leqslant q<p \leqslant \infty$ сплайнами со свободньми узлами и рациональными функциями.

Через $\Sigma_{r, n}(I)$, где $r, n \in \mathbb{N}$, обозначим множество всех определенных на промежутке $I$ кусочно полиномиальных сплайнов $\sigma_{r, n}$ порядка $\leqslant r$ с не более чем $n-1$ свободньми узлами. По определению $\sigma_{r, n} \in \Sigma_{r, n}(I)$, если сушествует некоторый набор $\left\{I_{i}\right\}_{i=1}^{m}$ из $m \leqslant n$ попарно непересекаюшихся интервалов $I_{i} \subseteq I$, суммарная длина которых равна длине $I$ и таких, что на каж дом из интервалов $I_{i}$ сплайн $\sigma_{r, n}$ совпадает с некоторым многочленом $\pi_{r}\left(\cdot ; I_{i}\right) \in P_{r}\left(I_{i}\right)$. Точки промежутка $I$, в которых сплайн $\sigma_{r, n}$ или хотя бы одна из его производных имеет разрыв, называются узлами. Узлы, лежащие внутри промежутка $I$, будем называть свободными.

Прежде чем сформулировать полученные результаты, условимся еше о некоторых обозначениях. Через $|I|$ будут обозначаться длины промежутков $I$, а через $c:=c(\alpha, \beta, \ldots, \gamma)$ - различные положительные "постоянные", зависящие от параметров $\alpha, \beta, \ldots, \gamma$. Если заданы две последовательности $\left\{a_{n}\right\}$ и $\left\{b_{n}\right\}$ положительных чисел $a_{n}$ и $b_{n}$, то эти последовательности удовлетворяют соотношению $a_{n} \asymp b_{n}, n \geqslant 1$, тогда и только тогда, когда сушествуют не зависяшие от $n$ постоянные $c_{1}>0$ и $c_{2}>0$ такие, что $c_{1} \leqslant a_{n} / b_{n} \leqslant c_{2}$ для всех $n \geqslant 1$.

Основные результаты настоящей работы приведены в следующих двух теоремах, где предполагается, что классы $\Delta_{+}^{s} B_{p}$ заданы на интервале $I:=(-1,1)$.

Tеорема 1. Eсли $r, s \in \mathbb{N} u 1 \leqslant q<p \leqslant \infty, m o$

$$
E\left(\Delta_{+}^{s} B_{p}, \Sigma_{r, n}\right)_{L_{q}} \asymp n^{-\min \{r, s\}}, \quad n \geqslant 1 .
$$

Teоpema 2. Ecлu $s \in \mathbb{N} u 1 \leqslant q<p \leqslant \infty$, mo

$$
E\left(\Delta_{+}^{s} B_{p}, R_{n}\right)_{L_{q}} \asymp n^{-s}, \quad n \geqslant 1 .
$$

\section{§2. Вспомогательные утверждения}

Приведенная ниже лемма 3 доказана в [14; лемма 3]. В этой лемме и далее для функций $x: I \rightarrow \mathbb{R}$, имеющих в точке $t \in I$ интервала $I$ конечные односторонние производные $x_{-}^{(k)}(t)$ и $x_{+}^{(k)}(t)$ порядка $k \in \mathbb{N}$, полагаем

$$
x^{(k)}(t):=\frac{1}{2}\left(x_{-}^{(k)}(t)+x_{+}^{(k)}(t)\right) .
$$

Ясно, что если в какой-либо точке интервала $I$ существует обычная производная порядка $k \in \mathbb{N}$, то она совпадает с так определяемой обобщенной производной. При $k=0$ полагаем $x^{(0)}(t):=x(t), t \in I$.

Лемма 3. Пусть $x \in \Delta_{+}^{s} L_{p}(I), s \in \mathbb{N}, 1 \leqslant p \leqslant \infty$, где $I$ - конечный интервал. Пусть $\bar{t}-$ середина интервала $I$ и

$$
\pi_{s}(t ; x ; \bar{t}):=\sum_{k=0}^{s-1} \frac{x^{(k)}(\bar{t})}{k !}(t-\bar{t})^{k}, \quad t \in I .
$$


Тогда для функиии

$$
\widetilde{x}(t):=x(t)-\pi_{s}(t ; x ; \bar{t}), \quad t \in I
$$

будет справедливо неравенство

$$
\|\widetilde{x}\|_{L_{p}(I)} \leqslant \widetilde{c}\|x\|_{L_{p}(I)},
$$

əде $\widetilde{c}=\widetilde{c}(s, p)$.

Лемма 4. Пусть $n \in \mathbb{N}, \gamma>0 u \delta_{i}>0, i=1, \ldots, n$. Тогда

$$
\sum_{i=1}^{n} \delta_{i}\left(\sum_{j=1}^{i} \delta_{j}\right)^{\gamma} \geqslant(\gamma+1)^{-1}\left(\sum_{i=1}^{n} \delta_{i}\right)^{\gamma+1}
$$

ДокАЗАТЕЛЬСТво. Если положить

$$
\tau_{0}:=0, \quad \tau_{i}:=\sum_{j=1}^{i} \delta_{j}, \quad i=1, \ldots, n,
$$

то утверждение леммы 4 следует из соотношений

$$
\begin{aligned}
\sum_{i=1}^{n} \delta_{i}\left(\sum_{j=1}^{i} \delta_{j}\right)^{\gamma} & =\sum_{i=1}^{n}\left(\tau_{i}-\tau_{i-1}\right) \tau_{i}^{\gamma} \\
& \geqslant \int_{\tau_{0}}^{\tau_{n}} \tau^{\gamma} d \tau=(\gamma+1)^{-1} \tau_{n}^{\gamma}=(\gamma+1)^{-1}\left(\sum_{i=1}^{n} \delta_{i}\right)^{\gamma+1}
\end{aligned}
$$

Лемма 4 доказана.

В следуюшей лемме и далее считаем, что $\left(\sum_{i=1}^{n}\left|a_{i}\right|^{\infty}\right)^{1 / \infty}:=\max _{1 \leqslant i \leqslant n}\left|a_{i}\right|$.

Лемма 5. Пусть $n \in \mathbb{N}, \delta_{i}>0, i=1, \ldots, n, 1 \leqslant p \leqslant \infty$, а числа $\alpha>0 u$ $\beta \geqslant 0$ удовлетворяют условию $\alpha-\beta-1 / p>0$. Тогда

$$
\left(\sum_{i=1}^{n}\left(\delta_{i}^{1 / p} \sum_{j=1}^{i} \delta_{j}^{-\alpha}\left(\sum_{k=j}^{i} \delta_{k}\right)^{\beta}\right)^{p}\right)^{-1 / p} \leqslant c\left(\sum_{i=1}^{n} \delta_{i}\right)^{\alpha-\beta-1 / p} n^{-\alpha-1}
$$

əде $c=c(\alpha, \beta, p)$.

ДоказАтельство. Рассмотрим вначале случай $1 \leqslant p<\infty$. Воспользовавшись известным неравенством

$$
\sum_{i=1}^{n}\left|a_{i}\right| \leqslant n^{1-1 / p}\left(\sum_{i=1}^{n}\left|a_{i}\right|^{p}\right)^{1 / p}
$$


получим

$$
\begin{aligned}
\left(\sum_{i=1}^{n}\left(\delta_{i}^{1 / p} \sum_{j=1}^{i} \delta_{j}^{-\alpha}\left(\sum_{k=j}^{i} \delta_{k}\right)^{\beta}\right)^{p}\right)^{-1 / p} & \\
& \leqslant n^{1-1 / p}\left(\sum_{i=1}^{n} \delta_{i}^{1 / p} \sum_{j=1}^{i} \delta_{j}^{-\alpha}\left(\sum_{k=j}^{i} \delta_{k}\right)^{\beta}\right)^{-1}
\end{aligned}
$$

Меняя затем порядок суммирования, имеем

$$
\sum_{i=1}^{n} \delta_{i}^{1 / p} \sum_{j=1}^{i} \delta_{j}^{-\alpha}\left(\sum_{k=j}^{i} \delta_{k}\right)^{\beta}=\sum_{i=1}^{n} \delta_{i}^{-\alpha} \sum_{j=i}^{n} \delta_{j}^{1 / p}\left(\sum_{k=i}^{j} \delta_{k}\right)^{\beta}
$$

Кроме того, учитывая соотношения

$$
\sum_{k=i}^{j} \delta_{k}=\left(\left(\sum_{k=i}^{j}\left(\delta_{k}^{1 / p}\right)^{p}\right)^{1 / p}\right)^{p} \geqslant n^{1-p}\left(\sum_{k=i}^{j} \delta_{k}^{1 / p}\right)^{p}
$$

приходим к неравенствам

$$
\sum_{i=1}^{n} \delta_{i}^{-\alpha} \sum_{j=i}^{n} \delta_{j}^{1 / p}\left(\sum_{k=i}^{j} \delta_{k}\right)^{\beta} \geqslant n^{\beta(1-p)} \sum_{i=1}^{n} \delta_{i}^{-\alpha} \sum_{j=i}^{n} \delta_{j}^{1 / p}\left(\sum_{k=i}^{j} \delta_{k}^{1 / p}\right)^{\beta p}
$$

Воспользовавшись леммой 4 при $\gamma:=\beta p$, получим

$$
\sum_{j=i}^{n} \delta_{j}^{1 / p}\left(\sum_{k=i}^{j} \delta_{k}^{1 / p}\right)^{\beta p} \geqslant(\beta p+1)^{-1}\left(\sum_{k=i}^{j} \delta_{k}^{1 / p}\right)^{\beta p+1}
$$

Следовательно,

$$
\sum_{i=1}^{n} \delta_{i}^{-\alpha} \sum_{j=i}^{n} \delta_{j}^{1 / p}\left(\sum_{k=i}^{j} \delta_{k}^{1 / p}\right)^{\beta p} \geqslant(\beta p+1)^{-1} \sum_{i=1}^{n} \delta_{i}^{-\alpha}\left(\sum_{j=i}^{n} \delta_{j}^{1 / p}\right)^{\beta p+1}
$$

Очевидно, что

$$
\begin{aligned}
&\left(\sum_{i=1}^{n} \delta_{i}^{-\alpha}\left(\sum_{j=i}^{n} \delta_{j}^{1 / p}\right)^{\beta p+1}\right)^{-1 /(\alpha p)} \\
&=\left(\sum_{i=1}^{n}\left(\delta_{i}^{1 / p}\left(\sum_{j=i}^{n} \delta_{j}^{1 / p}\right)^{-(\beta p+1) /(\alpha p)}\right)^{-\alpha p}\right)^{-1 /(\alpha p)}
\end{aligned}
$$

Воспользуемся теперь известным неравенством (см. [15; раздел 2.8 , неравенство (2.8.4)])

$$
\left(\frac{1}{n} \sum_{i=1}^{n} a_{i}^{-\lambda}\right)^{-1 / \lambda} \leqslant \frac{1}{n} \sum_{i=1}^{n} a_{i}
$$


которое справедливо для произвольных положительных чисел $\lambda_{\text {и }} a_{i}, i=1, \ldots, n$. Полагая в этом неравенстве $\lambda:=\alpha p$ и

$$
a_{i}:=\delta_{i}^{1 / p}\left(\sum_{j=i}^{n} \delta_{j}^{1 / p}\right)^{-(\beta p+1) /(\alpha p)}, \quad i=1, \ldots, n
$$

получаем оценку

$$
\begin{gathered}
\left(\sum_{i=1}^{n}\left(\delta_{i}^{1 / p}\left(\sum_{j=i}^{n} \delta_{j}^{1 / p}\right)^{-(\beta p+1) /(\alpha p)}\right)^{-\alpha p}\right)^{-1 /(\alpha p)} \\
\leqslant n^{-1-1 /(\alpha p)} \sum_{i=1}^{n} \delta_{i}^{1 / p}\left(\sum_{j=i}^{n} \delta_{j}^{1 / p}\right)^{-(\beta p+1) /(\alpha p)}
\end{gathered}
$$

Полагая затем

$$
\tau_{i}:=\sum_{j=i}^{n} \delta_{j}^{1 / p}, \quad i=1, \ldots, n, \quad \tau_{n+1}:=0
$$

имеем

$$
\begin{gathered}
\sum_{i=1}^{n} \delta_{i}^{1 / p}\left(\sum_{j=i}^{n} \delta_{j}^{1 / p}\right)^{-(\beta p+1) /(\alpha p)}=\sum_{i=1}^{n}\left(\tau_{i}-\tau_{i+1}\right) \tau_{i}^{-(\beta p+1) /(\alpha p)} \\
\leqslant \int_{\tau_{n+1}}^{\tau_{1}} \tau^{-(\beta p+1) /(\alpha p)} d \tau=\left(1-\frac{\beta p+1}{\alpha p}\right)^{-1} \tau_{1}^{1-(\beta p+1) /(\alpha p)} \\
=\frac{\alpha}{\alpha-\beta-1 / p}\left(\sum_{i=1}^{n} \delta_{i}^{1 / p}\right)^{(\alpha-\beta-1 / p) / \alpha}
\end{gathered}
$$

А так как

$$
\sum_{i=1}^{n} \delta_{i}^{1 / p} \leqslant n^{1-1 / p}\left(\sum_{i=1}^{n} \delta_{i}\right)^{1 / p}
$$

то из соотношений $(2.1)-(2.5)$ и (2.7)-(2.9) следует утверждение леммы 5 при $1 \leqslant$ $p<\infty$, где $c:=(\beta p+1) \alpha^{\alpha p}(\alpha-\beta-1 / p)^{-\alpha p}$.

Осталось рассмотреть случай $p=\infty$. В этом случае необходимо доказать, что

$$
\left(\sum_{i=1}^{n} \delta_{i}^{-\alpha}\left(\sum_{j=i}^{n} \delta_{j}\right)^{\beta}\right)^{-1} \leqslant c\left(\sum_{i=1}^{n} \delta_{i}\right)^{\alpha-\beta} n^{-\alpha-1}
$$

где $c=c(\alpha, \beta)$.

Вновь воспользовавшись неравенством (2.6), но уже для $\lambda:=\alpha$ и

$$
a_{i}:=\delta_{i}\left(\sum_{j=i}^{n} \delta_{j}\right)^{-\beta / \alpha}, \quad i=1, \ldots, n
$$


имеем

$$
\left(\sum_{i=1}^{n}\left(\delta_{i}\left(\sum_{j=i}^{n} \delta_{j}\right)^{-\beta / \alpha}\right)^{-\alpha}\right)^{-1 / \alpha} \leqslant n^{-1-1 / \alpha} \sum_{i=1}^{n} \delta_{i}\left(\sum_{j=i}^{n} \delta_{j}\right)^{-\beta / \alpha} .
$$

Далее, полагая

$$
\tau_{i}:=\sum_{j=i}^{n} \delta_{j}, \quad i=1, \ldots, n, \quad \tau_{n+1}:=0
$$

получаем

$$
\begin{aligned}
\sum_{i=1}^{n} \delta_{i}\left(\sum_{j=i}^{n} \delta_{j}\right)^{-\beta / \alpha}=\sum_{i=1}^{n}\left(\tau_{i}-\tau_{i+1}\right) \tau_{i}^{-\beta / \alpha} \leqslant \sum_{i=1}^{n} \int_{\tau_{i+1}}^{\tau_{i}} \tau^{-\beta / \alpha} d \tau \\
=\int_{\tau_{n+1}}^{\tau_{1}} \tau^{-\beta / \alpha} d \tau=\left(1-\frac{\beta}{\alpha}\right)^{-1} \tau_{1}^{1-\beta / \alpha}=\left(1-\frac{\beta}{\alpha}\right)^{-1}\left(\sum_{i=1}^{n} \delta_{i}\right)^{1-\beta / \alpha}
\end{aligned}
$$

Но тогда из (2.11) и (2.12) следует неравенство $(2.10)$, где $c:=(\alpha-\beta)^{-\alpha} \alpha^{\alpha}$. Лемма 5 доказана.

ЛЕмма 6. Пусть на множестве всех отрезков $[a, b] \subseteq[0,1]$, где $a \leqslant b$, определено отображсение $\mu:[a, b] \rightarrow \mathbb{R}$ такое, что

1) $\mu([a, b]) \geqslant 0 \quad \forall[a, b] \subseteq[0,1]$;

2) $\mu([c, c])=0 \quad \forall c \in[0,1]$;

3) $\mu([a, t])$ - непрерывная и неубьвающая функция по $t$ на $[a, 1] \forall a \in[0,1]$;

4) $\mu([t, b])$ - непрерьвная и невозрастающая функиия по $t$ на $[0, b] \forall b \in[0,1]$.

Тогда при каждом $n \in \mathbb{N}$ существует разбиение $0=: t_{0}^{*} \leqslant t_{1}^{*} \leqslant \cdots \leqslant t_{n}^{*}:=1$ отрезка $[0,1]$ на отрезки $\left[t_{i-1}^{*}, t_{i}^{*}\right], i=1, \ldots, n$, такие, что

$$
\max _{1 \leqslant i \leqslant n} \mu\left(\left[t_{i-1}^{*}, t_{i}^{*}\right]\right)=\min _{1 \leqslant i \leqslant n} \mu\left(\left[t_{i-1}^{*}, t_{i}^{*}\right]\right)
$$

ДоКАЗАТЕЛЬСТво. При $n=1$ утверждение леммы 6 очевидно. Поэтому будем считать, что $n>1$. Полагая $t_{0}:=0$ и $t_{n}:=1$, определяем на $(n-1)$-мерном симплексе

$$
S^{n-1}:=\left\{\mathbf{t} \mid \mathbf{t}:=\left(t_{1}, \ldots, t_{n-1}\right), 0 \leqslant t_{1} \leqslant \cdots \leqslant t_{n-1} \leqslant 1\right\}
$$

следующие непрерьвные функции

$$
\begin{gathered}
M(\mathbf{t}):=\max _{1 \leqslant i \leqslant n} \mu\left(\left[t_{i-1}, t_{i}\right]\right), \quad m(\mathbf{t}):=\min _{1 \leqslant i \leqslant n} \mu\left(\left[t_{i-1}, t_{i}\right]\right) \\
\Delta(\mathbf{t}):=M(\mathbf{t})-m(\mathbf{t}) .
\end{gathered}
$$

Обозначим через $J(\mathbf{t}):=\{j(\mathbf{t})\}$ множество всех индексов $j(\mathbf{t}) \in\{1, \ldots, n\}$ таких, что

$$
\mu\left(\left[t_{j(\mathbf{t})-1}, t_{j(\mathbf{t})}\right]\right)=M(\mathbf{t}) .
$$


Пусть $T^{*}$ - множество всех точек $\mathbf{t}^{*}:=\left(t_{1}^{*}, \ldots, t_{n-1}^{*}\right) \in S^{n-1}$ таких, что

$$
\Delta\left(\mathbf{t}^{*}\right)=\min _{\mathbf{t} \in S^{n-1}} \Delta(\mathbf{t})
$$

Так как функция $\Delta$ непрерывна, то $T^{*} \neq \varnothing$. Зафиксируем произвольную точку $\mathbf{t}^{*} \in T^{*}$ и допустим от противного, что $\Delta\left(\mathbf{t}^{*}\right)>0$. Тогда $M\left(\mathbf{t}^{*}\right)>0$ и $1 \leqslant \operatorname{card} J\left(\mathbf{t}^{*}\right)<n$. Также ясно, что сушествует индекс $j\left(\mathbf{t}^{*}\right) \in J\left(\mathbf{t}^{*}\right)$ такой, что хотя бы один из индексов $j\left(\mathbf{t}^{*}\right)-1$ или $j\left(\mathbf{t}^{*}\right)+1$ не принадлежит множеству $J\left(\mathbf{t}^{*}\right)$. Не ограничивая общности рассуждений, будем считать, что $1<$ $j\left(\mathbf{t}^{*}\right) \leqslant n$ и $\left(j\left(\mathbf{t}^{*}\right)-1\right) \notin J\left(\mathbf{t}^{*}\right)$. Очевидно, что в этом случае $t_{j\left(\mathbf{t}^{*}\right)-1}^{*}<t_{j\left(\mathbf{t}^{*}\right)}^{*}$ и $\mu\left(\left[t_{j\left(\mathbf{t}^{*}\right)-2}^{*}, t_{j\left(\mathbf{t}^{*}\right)-1}^{*}\right]\right)<\mu\left(\left[t_{j\left(\mathbf{t}^{*}\right)-1}^{*}, t_{j\left(\mathbf{t}^{*}\right)}^{*}\right]\right)$. Но тогда из условий 1$\left.)-4\right)$ леммы 6 следует существование числа $\widehat{t}^{*} \in\left(t_{j\left(\mathbf{t}^{*}\right)-1}^{*}, t_{j\left(\mathbf{t}^{*}\right)}^{*}\right)$ такого, что

$$
m\left(\mathbf{t}^{*}\right)<\mu\left(\left[t_{j\left(\mathbf{t}^{*}\right)-2}^{*}, \widehat{t}^{*}\right]\right)<\mu\left(\left[\widehat{t}^{*}, t_{j\left(\mathbf{t}^{*}\right)}^{*}\right]\right)<M\left(\mathbf{t}^{*}\right) .
$$

Полагаем $\widehat{\mathbf{t}}^{*}=\left(\widehat{t}_{1}^{*}, \ldots, \widehat{t}_{n-1}^{*}\right)$, где $\widehat{t}_{i}^{*}:=t_{i}^{*}$ при $i \neq j\left(\mathbf{t}^{*}\right)-1$, a $\widehat{t}_{j\left(\mathbf{t}^{*}\right)-1}^{*}=\widehat{t}^{*}$.

Ясно, что если card $J\left(\mathbf{t}^{*}\right)=1$, то $\Delta\left(\widehat{\mathbf{t}}^{*}\right)<\Delta\left(\mathbf{t}^{*}\right)$ и мы приходим к неравенству

$$
\min _{\mathbf{t} \in S^{n-1}} \Delta(\mathbf{t}) \leqslant \Delta\left(\widehat{\mathbf{t}}^{*}\right)<\min _{\mathbf{t} \in S^{n-1}} \Delta(\mathbf{t}),
$$

которое свидетельствует о невозможности выполнения неравенства $\Delta\left(\mathbf{t}^{*}\right)>0$.

Если же card $J\left(\mathbf{t}^{*}\right)>1$, то $\widehat{\mathbf{t}}^{*} \in T^{*}$ и при этом card $J\left(\widehat{\mathbf{t}}^{*}\right)=\operatorname{card} J\left(\mathbf{t}^{*}\right)-1$.

Повторяя конечное число раз приведенные выше рассуждения, мы докажем существование точки $\check{\mathbf{t}}^{*} \in S^{n-1}$ такой, что

$$
\min _{\mathbf{t} \in S^{n-1}} \Delta(\mathbf{t}) \leqslant \Delta\left(\check{\mathbf{t}}^{*}\right)<\min _{\mathbf{t} \in S^{n-1}} \Delta(\mathbf{t}) .
$$

Полученное противоречие свидетельствует о том, что для $\mathbf{t}^{*} \in T^{*}$ будет выполняться равенство $\Delta\left(\mathbf{t}^{*}\right)=0$. Лемма 6 доказана.

ЛЕмма 7. Пусть $I_{*}$ и $I^{*}-$ конечные интервалы такие, что $I_{*} \subseteq I^{*}$. Если $r \in \mathbb{N}_{0}, s \in \mathbb{N}, r \leqslant s-1 u 1 \leqslant p, q \leqslant \infty$, то для каждого многочлена $\pi_{s}$ порядка $\leqslant s$ справедливо неравенство

$$
\left\|\pi_{s}^{(r)}\right\|_{L_{q}\left(I^{*}\right)} \leqslant c\left|I_{*}\right|^{-s+1 / p^{\prime}}\left|I^{*}\right|^{s-r-1 / q^{\prime}}\left\|\pi_{s}\right\|_{L_{p}\left(I_{*}\right)},
$$

где $c=c(r, s), 1 / p^{\prime}:=1-1 / p$ u $1 / q^{\prime}:=1-1 / q$.

ДоКАЗАТЕЛЬСТво. Не ограничивая общности рассуждений, будем предполагать, что центр интервала $I_{*}$ находится в точке $\bar{t}_{*}=0$. Полагаем

$$
\phi(t):= \begin{cases}\gamma^{-1} e^{t^{2} /\left(t^{2}-1\right)}, & |t|<1, \\ 0, & |t| \geqslant 1,\end{cases}
$$

где

$$
\gamma:=\int_{-1}^{1} e^{t^{2} /\left(t^{2}-1\right)} d t
$$


Ясно, что $\phi \in C_{0}^{\infty}(\mathbb{R})$ и $\|\phi\|_{L_{1}(-1,1)}=1$. Отправляясь от функции $\phi$, рассмотрим следующую функцию

$$
\phi\left(t ; I_{*}\right):=\frac{2}{\left|I_{*}\right|} \phi\left(\frac{2 t}{\left|I_{*}\right|}\right), \quad t \in \mathbb{R} .
$$

Очевидно, что $\phi\left(t ; I_{*}\right)>0$ для $t \in I_{*}$ и $\phi\left(t ; I_{*}\right)=0$ для $t \in \mathbb{R} \backslash I_{*}$. Также очевидно, что $\left\|\phi\left(\cdot ; I_{*}\right)\right\|_{L_{1}\left(I_{*}\right)}=1$. Нетрудно проверить, что

$$
\left\|\phi^{(l)}\left(\cdot ; I_{*}\right)\right\|_{L_{\infty}\left(I_{*}\right)} \leqslant c_{l}\left|I_{*}\right|^{-l-1}, \quad l \in \mathbb{N}_{0},
$$

где $c_{k}$ зависят только от $k$.

Из формулы Тейлора следует, что для каждого многочлена $\pi_{s}$ справедливо тождество

$$
\pi_{s}^{(r)}(t)=\sum_{k=r}^{s-1} \frac{\pi_{s}^{(k)}(\tau)}{(k-r) !}(t-\tau)^{k-r}, \quad t, \tau \in \mathbb{R}, \quad r \in \mathbb{N}_{0} .
$$

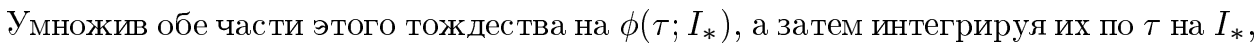
получаем

$$
\pi_{s}^{(r)}(t)=\sum_{k=r}^{s-1} \frac{1}{(k-r) !} \int_{I_{*}} \pi_{s}^{(k)}(\tau)(t-\tau)^{k-r} \phi\left(\tau ; I_{*}\right) d \tau .
$$

При этом было использовано равенство $\left\|\phi\left(\cdot ; I_{*}\right)\right\|_{L_{1}\left(I_{*}\right)}=1$. Далее, с помощью интегрирования по частям приходим к равенствам

$$
\int_{I_{*}} \pi_{s}^{(k)}(\tau)(t-\tau)^{k-r} \phi\left(\tau ; I_{*}\right) d \tau=(-1)^{k} \int_{I_{*}} \pi_{s}(\tau) \frac{d^{k}}{d \tau^{k}}\left((t-\tau)^{k-r} \phi\left(\tau ; I_{*}\right)\right) d \tau .
$$

Воспользовавшись формулой Лейбница для производных произведения двух функций, имеем

$$
\begin{gathered}
\frac{d^{k}}{d \tau^{k}}\left((t-\tau)^{k-r} \phi\left(\tau ; I_{*}\right)\right)=\sum_{l=0}^{k}\left(\begin{array}{c}
k \\
l
\end{array}\right)\left(\frac{d^{k-l}}{d \tau^{k-l}}(t-\tau)^{k-r}\right) \phi^{(l)}\left(\tau ; I_{*}\right) \\
=\sum_{l=r}^{k}(-1)^{k-l}\left(\begin{array}{l}
k \\
l
\end{array}\right) \frac{(k-r) !}{(l-r) !}(t-\tau)^{l-r} \phi^{(l)}\left(\tau ; I_{*}\right) .
\end{gathered}
$$

Но тогда из (2.14)-(2.16) следует тождество

$$
\pi_{s}^{(r)}(t)=\sum_{k=r}^{s-1} \sum_{l=r}^{k} \frac{(-1)^{l}}{(l-r) !}\left(\begin{array}{l}
k \\
l
\end{array}\right) \int_{I_{*}} \pi_{s}(\tau)(t-\tau)^{l-r} \phi^{(l)}\left(\tau ; I_{*}\right) d \tau, \quad t \in \mathbb{R} .
$$

А так как $|t-\tau| \leqslant\left|I^{*}\right|$ для всех $t \in I^{*}$ и $\tau \in I_{*}$, то из (2.17) с учетом неравенства (2.13) следует оценка

$$
\left|\pi_{s}^{(r)}(t)\right| \leqslant\left(\sum_{k=r}^{s-1} \sum_{l=r}^{k} \frac{c_{l}}{(l-r) !}\left(\begin{array}{l}
k \\
l
\end{array}\right) \frac{\left|I^{*}\right|^{l-r}}{\left|I_{*}\right|^{l+1}}\right)\left\|\pi_{s}\right\|_{L_{1}\left(I_{*}\right)}, \quad t \in I^{*} .
$$


Кроме того,

$$
\begin{gathered}
\sum_{k=r}^{s-1} \sum_{l=r}^{k} \frac{c_{l}}{(l-r) !}\left(\begin{array}{c}
k \\
l
\end{array}\right) \frac{\left|I^{*}\right|^{l-r}}{\left|I_{*}\right|^{l+1}} \leqslant\left(\max _{r \leqslant l \leqslant s-1} c_{l}\right) \frac{1}{\left|I^{*}\right|^{r}\left|I_{*}\right|} \sum_{k=r}^{s-1} \sum_{l=0}^{k}\left(\begin{array}{c}
k \\
l
\end{array}\right)\left(\frac{\left|I^{*}\right|}{\left|I_{*}\right|}\right)^{l} \\
=\left(\max _{r \leqslant l \leqslant s-1} c_{l}\right) \frac{1}{\left|I^{*}\right| r\left|I_{*}\right|} \sum_{k=r}^{s-1}\left(1+\frac{\left|I^{*}\right|}{\left|I_{*}\right|}\right)^{k} \\
\leqslant(s-r) 2^{s-1}\left(\max _{r \leqslant l \leqslant s-1} c_{l}\right) \frac{\left|I^{*}\right|^{s-r-1}}{\left|I_{*}\right|^{s}} .
\end{gathered}
$$

Поэтому из (2.18) легко получить оценку

$$
\left\|\pi_{s}^{(r)}\right\|_{L_{q}\left(I^{*}\right)} \leqslant(s-r) 2^{s-1}\left(\max _{r \leqslant l \leqslant s-1} c_{l}\right)\left|I_{*}\right|^{-s}\left|I^{*}\right|^{s-r-1 / q^{\prime}}\left\|\pi_{s}\right\|_{L_{1}\left(I_{*}\right)}
$$

А из этой оценки в силу неравенства Гёльдера следует неравенство

$$
\left\|\pi_{s}\right\|_{L_{q}\left(I^{*}\right)} \leqslant(s-r) 2^{s-1}\left(\max _{r \leqslant l \leqslant s-1} c_{l}\right)\left|I_{*}\right|^{-s+1 / p^{\prime}}\left|I^{*}\right|^{s-r-1 / q^{\prime}}\left\|\pi_{s}\right\|_{L_{p}\left(I_{*}\right)} .
$$

Таким образом, лемма 7 доказана.

Обозначим через

$$
E\left(x, R_{n}\right)_{L_{q}}:=\inf _{\rho_{n} \in R_{n}}\left\|x-\rho_{n}\right\|_{L_{q}}
$$

наилучшее приближение функции $x$ в метрике $L_{q}, 1 \leqslant q \leqslant \infty$, на интервале $I:=$ $(-1,1)$ рациональными функциями из $R_{n}$, а через

$$
E\left(x, \Sigma_{r, n}\right)_{L_{q}}:=\inf _{\sigma_{r, n} \in \Sigma_{r, n}}\left\|x-\sigma_{r, n}\right\|_{L_{q}}
$$

- наилучшее приближение в $L_{q}$ этой же функции сплайнами из $\Sigma_{r, n}$.

В следуюшей лемме приводится лишь необходимая нам часть более обших результатов, установленных в [16] и [17].

Лемма 8. Пусть $I:=(-1,1), r, n \in \mathbb{N}, 1 \leqslant q<\infty u \lambda>0$. Тогда для каждой функиии $x \in L_{q}(I)$ справедливы неравенства

$$
E\left(x, R_{n}\right)_{L_{q}} \leqslant c n^{-\lambda} \sum_{m=1}^{n} m^{\lambda-1} E\left(x, \Sigma_{r, m}\right)_{L_{q}}, \quad n \geqslant r
$$

əде $c=c(r, \lambda, q)$. 


\section{§ 3. Доказательство оценок сверху в теореме 1}

При доказательстве оценок сверху будем вначале предполагать, что $x \in \Delta_{+}^{s} C^{s-1}(\bar{I})$, где $\bar{I}:=[-1,1]$. То есть предполагаем, что функции $x$ являются $s$-монотонными на отрезке $\bar{I}$, а их (обычные) производные $x^{(s-1)}$ непрерывны на $\bar{I}$. Кроме того, будем предполагать, что эти функции удовлетворяют дополнительному условию

$$
x^{(k)}(0)=0, \quad k=0, \ldots, s-1 .
$$

Нетрудно убедиться, что при этом условии для всех $k=0, \ldots, s-1$ выполняются неравенства $x^{(k)}(t) \geqslant 0, t \in \bar{I}_{+}:=[0,1]$. Если же $t \in \bar{I}_{-}:=[-1,0]$, то $(-1)^{s-k} x^{(k)}(t) \geqslant 0, k=0, \ldots, s-1$. Отметим, что при условии (3.1) все производные $x^{(k)}, k=0, \ldots, s-1$, являются неубывающими на $\bar{I}_{+}$функциями. На отрезке же $\bar{I}_{-}$происходит чередование монотонности для производных $x^{(k)}$, $k=0, \ldots, s-1$.

Зафиксировав $r \leqslant s$, построим на каждом из отрезков $\bar{I}_{+}$и $\bar{I}_{-}$приближаюшие сплайны $\sigma_{r, n}\left(\cdot ; x ; \bar{I}_{+}\right) \in \Sigma_{r, n}\left(\bar{I}_{+}\right)$и $\sigma_{r, n}\left(\cdot ; x ; \bar{I}_{-}\right) \in \Sigma_{r, n}\left(\bar{I}_{-}\right)$. Построение приближающих сплайнов и оценки приближения осуществим подробно лишш для отрезка $\bar{I}_{+}$, так как для отрезка $\bar{I}_{-}$все рассуждения являются аналогичными.

Если $n=1$, то полагаем

$$
\sigma_{r, 1}\left(t ; x ; \bar{I}_{+}\right):=0, \quad t \in \bar{I}_{+} .
$$

Очевидно, что из неравенства Гёльдера следует оценка

$$
\left\|x(\cdot)-\sigma_{r, 1}\left(\cdot ; x ; \bar{I}_{+}\right)\right\|_{L_{q}\left(\bar{I}_{+}\right)} \leqslant\|x\|_{L_{p}\left(\bar{I}_{+}\right)}, \quad 1 \leqslant q<p \leqslant \infty .
$$

Если же $n>1$, то при фиксированных $r \in \mathbb{N}, 1 \leqslant q<\infty$ и $x$ определим на множестве всех отрезков $[a, b] \subseteq \bar{I}_{+}$, где $a \leqslant b$, отображения $\mu_{r, q}([a, b] ; x) \rightarrow \mathbb{R}$, полагая

$$
\mu_{1, q}([a, b] ; x):=\left(\int_{a}^{b}(x(t)-x(a))^{q} d t\right)^{1 / q}
$$

для $r=1$ и

$$
\mu_{r, q}([a, b] ; x):=\left(\int_{a}^{b}\left(\int_{a}^{t}\left(x^{(r-1)}(\tau)-x^{(r-1)}(a)\right)(t-\tau)^{r-2} d \tau\right)^{q} d t\right)^{1 / q}
$$

для $r>1$.

Очевидно, что эти отображения удовлетворяют условиям 1)-4) леммы 6 . Поэтому сушествуют (зависяшие от $r, q$ и $x$ ) разбиения $0=: t_{0}^{*} \leqslant t_{1}^{*} \leqslant \cdots \leqslant t_{n}^{*}:=1$ отрезка $\bar{I}_{+}$такие, что

$$
\max _{1 \leqslant i \leqslant n} \mu_{r, q}\left(\left[t_{i-1}^{*}, t_{i}^{*}\right] ; x\right)=\min _{1 \leqslant i \leqslant n} \mu_{r, q}\left(\left[t_{i-1}^{*}, t_{i}^{*}\right] ; x\right) .
$$

Если

$$
\max _{1 \leqslant i \leqslant n} \mu_{r, q}\left(\left[t_{i-1}^{*}, t_{i}^{*}\right] ; x\right)=\min _{1 \leqslant i \leqslant n} \mu_{r, q}\left(\left[t_{i-1}^{*}, t_{i}^{*}\right] ; x\right)=0,
$$


то из формулы Тейлора следует, что функция $x$ совпадает на $\bar{I}_{+}$с алгебраическим многочленом

$$
\pi_{r}\left(t ; x ; \bar{I}_{+}\right):=\sum_{k=0}^{r-1} \frac{x^{(k)}(0)}{k !} t^{k}, \quad t \in \bar{I}_{+} .
$$

Поэтому в случае выполнения условия (3.6) приближающий сплайн определяем, полагая

$$
\sigma_{r, n}\left(t ; x ; \bar{I}_{+}\right):=\pi_{r}\left(t ; x ; \bar{I}_{+}\right), \quad t \in \bar{I}_{+}
$$

При этом очевидно, что

$$
\left\|x(\cdot)-\sigma_{r, n}\left(\cdot ; x ; \bar{I}_{+}\right)\right\|_{L_{q}\left(\bar{I}_{+}\right)}=0
$$

Если же

$$
\max _{1 \leqslant i \leqslant n} \mu_{r, q}\left(\left[t_{i-1}^{*}, t_{i}^{*}\right] ; x\right)=\min _{1 \leqslant i \leqslant n} \mu_{r, q}\left(\left[t_{i-1}^{*}, t_{i}^{*}\right] ; x\right)>0
$$

то из условия 2) леммы 6 следует, что для соответствующего разбиения отрезка $\bar{I}_{+}$ будут выполняться неравенства $t_{0}^{*}<t_{1}^{*}<\cdots<t_{n}^{*}$. Полагая $I_{i}^{*}:=\left[t_{i-1}^{*}, t_{i}^{*}\right)$, $i=1, \ldots, n-1$, и $I_{n}^{*}:=\left[t_{n-1}^{*}, t_{n}^{*}\right]$, определяем на каждом из промежутков $I_{i}^{*}$ многочлены

$$
\pi_{r}\left(t ; x ; I_{i}^{*}\right):=\sum_{k=0}^{r-1} \frac{x^{(k)}\left(t_{i-1}^{*}\right)}{k !}\left(t-t_{i-1}^{*}\right)^{k}, \quad t \in I_{i}^{*}, \quad i=1, \ldots, n
$$

порядка $\leqslant r$. При условии (3.9) приближающий сплайн определяем, полагая

$$
\sigma_{r, n}\left(t ; x ; \bar{I}_{+}\right):=\pi_{r}\left(t ; x ; I_{i}^{*}\right), \quad t \in I_{i}^{*}, \quad i=1, \ldots, n
$$

Оценим уклонения этих сплайнов от функции $x$ в метрике $L_{q}\left(\bar{I}_{+}\right), 1 \leqslant q<$ $p \leqslant \infty$. Отметим, что при получении оценок будут использоваться монотонность и неотрицательность всех производных $x^{(k)}, k=0, \ldots, s-1$, а также условие (3.1).

Если $r=1$, то ясно, что

$$
\begin{aligned}
& \left\|x(\cdot)-\sigma_{1, n}\left(\cdot ; x ; \bar{I}_{+}\right)\right\|_{L_{q}\left(\bar{I}_{+}\right)}=\left(\sum_{i=1}^{n} \int_{I_{i}^{*}}\left(x(t)-\pi_{1}\left(t ; x ; I_{i}^{*}\right)\right)^{q} d t\right)^{1 / q} \\
& =\left(\sum_{i=1}^{n} \int_{I_{i}^{*}}\left(x(t)-x\left(t_{i-1}^{*}\right)\right)^{q} d t\right)^{1 / q} \\
& \quad \leqslant n^{1 / q} \max _{1 \leqslant i \leqslant n}\left(\int_{I_{i}^{*}}\left(x(t)-x\left(t_{i-1}^{*}\right)\right)^{q} d t\right)^{1 / q}=n^{1 / q} \max _{1 \leqslant i \leqslant n} \mu_{1, q}\left(\bar{I}_{i}^{*} ; x\right),
\end{aligned}
$$

где $\bar{I}_{i}^{*}:=\left[t_{i-1}^{*}, t_{i}^{*}\right], i=1, \ldots, n$. 
Теперь оценим снизу норму $\|x\|_{L_{p}\left(\bar{I}_{+}\right)}$через значения отображений вида $(3.4)$ и длины промежутков $I_{i}^{*}$. Учитьвая, что $x\left(t_{0}^{*}\right)=0$, имеем

$$
\begin{gathered}
x(t)=x(t)-x\left(t_{0}^{*}\right), \quad t \in I_{1}^{*}, \\
x(t)=\left(x(t)-x\left(t_{i-1}^{*}\right)\right)+\sum_{j=1}^{i-1}\left(x\left(t_{j}^{*}\right)-x\left(t_{j-1}^{*}\right)\right), \quad t \in I_{i}^{*}, \quad i=2, \ldots, n .
\end{gathered}
$$

Воспользовавшись неравенством Гёльдера, получим

$$
\left(\int_{I_{i}^{*}}\left(x(t)-x\left(t_{i-1}^{*}\right)\right)^{p} d t\right)^{1 / p} \geqslant\left|I_{i}^{*}\right|^{1 / p-1 / q} \mu_{1, q}\left(\bar{I}_{i}^{*} ; x\right), \quad i=1, \ldots, n .
$$

Кроме того, очевидно, что

$$
x\left(t_{j}^{*}\right)-x\left(t_{j-1}^{*}\right) \geqslant\left|I_{j}^{*}\right|^{-1 / q} \mu_{1, q}\left(\bar{I}_{j}^{*} ; x\right), \quad j=1, \ldots, i-1, \quad i=2, \ldots, n .
$$

Но тогда при $1 \leqslant p<\infty$ будут выполняться соотношения

$$
\begin{aligned}
\|x\|_{L_{p}\left(\bar{I}_{+}\right)}^{p}= & \int_{I_{1}^{*}}\left(x(t)-x\left(t_{0}^{*}\right)\right)^{p} d t \\
& +\sum_{i=2}^{n} \int_{I_{i}^{*}}\left(\left(x(t)-x\left(t_{i-1}^{*}\right)\right)+\sum_{j=1}^{i-1}\left(x\left(t_{j}^{*}\right)-x\left(t_{j-1}^{*}\right)\right)\right)^{p} d t \\
\geqslant & \sum_{i=1}^{n} \int_{I_{i}^{*}}\left(x(t)-x\left(t_{i-1}^{*}\right)\right)^{p} d t+\sum_{i=2}^{n} \int_{I_{i}^{*}}\left(\sum_{j=1}^{i-1}\left(x\left(t_{j}^{*}\right)-x\left(t_{j-1}^{*}\right)\right)\right)^{p} d t \\
\geqslant & \left(\sum_{i=1}^{n}\left|I_{i}^{*}\right|^{1-p / q}+\sum_{i=2}^{n}\left|I_{i}^{*}\right|\left(\sum_{j=1}^{i-1}\left|I_{j}^{*}\right|^{-1 / q}\right)^{p}\right)\left(\min _{1 \leqslant i \leqslant n} \mu_{1, q}\left(\bar{I}_{i}^{*} ; x\right)\right)^{p} \\
\geqslant & 2^{1-p}\left(\sum_{i=1}^{n}\left(\left|I_{i}^{*}\right|^{1 / p} \sum_{j=1}^{i}\left|I_{j}^{*}\right|^{-1 / q}\right)^{p}\right)\left(\min _{1 \leqslant i \leqslant n} \mu_{1, q}\left(\bar{I}_{i}^{*} ; x\right)\right)^{p} .
\end{aligned}
$$

Кроме неравенств (3.13) и (3.14) здесь использовались также неравенства

$$
(|a|+|b|)^{p} \geqslant|a|^{p}+|b|^{p} \geqslant 2^{1-p}(|a|+|b|)^{p} .
$$

Таким образом,

$$
\min _{1 \leqslant i \leqslant n} \mu_{1, q}\left(\bar{I}_{i}^{*} ; x\right) \leqslant c_{*}\|x\|_{L_{p}\left(\bar{I}_{+}\right)}\left(\sum_{i=1}^{n}\left(\left|I_{i}^{*}\right|^{1 / p} \sum_{j=1}^{i}\left|I_{j}^{*}\right|^{-1 / q}\right)^{p}\right)^{-1 / p},
$$

где $c_{*}=2^{1-1 / p}$. Нетрудно проверить, что эта оценка верна и в случае $p=\infty$. Поэтому при всех $1 \leqslant q<p \leqslant \infty$ из (3.12), (3.15) и (3.9) следует оценка сверху

$$
\begin{aligned}
& \left\|x(\cdot)-\sigma_{1, n}\left(\cdot ; x ; \bar{I}_{+}\right)\right\|_{L_{q}\left(\bar{I}_{+}\right)} \\
& \quad \leqslant c^{*}\|x\|_{L_{p}\left(\bar{I}_{+}\right)} n^{1 / q}\left(\sum_{i=1}^{n}\left(\left|I_{i}^{*}\right|^{1 / p} \sum_{j=1}^{i}\left|I_{j}^{*}\right|^{-1 / q}\right)^{p}\right)^{-1 / p},
\end{aligned}
$$

где $c^{*}=2^{1-1 / p}$. 
Рассмотрим теперь случай $r>1$. Из (3.11) следует, что

$$
\left\|x(\cdot)-\sigma_{r, n}\left(\cdot ; x ; \bar{I}_{+}\right)\right\|_{L_{q}\left(\bar{I}_{+}\right)}=\left(\sum_{i=1}^{n} \int_{I_{i}^{*}}\left(x(t)-\pi_{r}\left(t ; x ; I_{i}^{*}\right)\right)^{q} d t\right)^{1 / q}
$$

где многочлены $\pi_{r-1}\left(\cdot ; x ; I_{i}^{*}\right)$ определены в $(3.10)$.

Воспользовавшись формулой Тейлора, имеем

$$
x(t)-\pi_{r}\left(t ; x ; I_{i}^{*}\right)=\frac{1}{(r-2) !} \int_{t_{i-1}^{*}}^{t}\left(x^{(r-1)}(\tau)-x^{(r-1)}\left(t_{i-1}^{*}\right)\right)(t-\tau)^{r-2} d \tau, \quad t \in I_{i}^{*} .
$$

Но тогда

$$
\begin{aligned}
& \left(\sum_{i=1}^{n} \int_{I_{i}^{*}}\left(x(t)-\pi_{r}\left(t ; x ; I_{i}^{*}\right)\right)^{q} d t\right)^{1 / q} \\
& \quad \leqslant \frac{1}{(r-2) !}\left(\sum_{i=1}^{n} \int_{I_{i}^{*}}\left(\int_{t_{i-1}^{*}}^{t}\left(x^{(r-1)}(\tau)-x^{(r-1)}\left(t_{i-1}^{*}\right)\right)(t-\tau)^{r-2} d \tau\right)^{q} d t\right)^{1 / q} \\
& \quad \leqslant \frac{n^{1 / q}}{(r-2) !} \max _{1 \leqslant i \leqslant n} \mu_{r, q}\left(\bar{I}_{i}^{*} ; x\right) .
\end{aligned}
$$

Поэтому из (3.17) следует, что

$$
\left\|x(\cdot)-\sigma_{r, n}\left(\cdot ; x ; \bar{I}_{+}\right)\right\|_{L_{q}\left(\bar{I}_{+}\right)} \leqslant \frac{n^{1 / q}}{(r-2) !} \max _{1 \leqslant i \leqslant n} \mu_{r, q}\left(\bar{I}_{i}^{*} ; x\right)
$$

Оценим теперь снизу норму $\|x\|_{L_{p}\left(\bar{I}_{+}\right)}$через значения отображений вида $(3.5)$ и длины промежутков $I_{i}^{*}$. В силу условия (3.1) имеем

$$
\begin{gathered}
x(t)=x(t)-\pi_{r}\left(t ; x ; I_{1}^{*}\right), \quad t \in I_{1}^{*}, \\
x(t)=x(t)-\pi_{r}\left(t ; x ; I_{i}^{*}\right)+\sum_{j=2}^{i}\left(\pi_{r}\left(t ; x ; I_{j}^{*}\right)-\pi_{r}\left(t ; x ; I_{j-1}^{*}\right)\right), \quad t \in I_{i}^{*}, \quad i=2, \ldots, n .
\end{gathered}
$$

Воспользовавшись этими равенствами и неравенством $|a|+|b| \geqslant\left(|a|^{p}+|b|^{p}\right)^{1 / p}$, при $1 \leqslant p<\infty$ получим

$$
\begin{aligned}
\|x\|_{L_{p}\left(I_{+}\right)}^{p} \geqslant & \sum_{i=1}^{n} \int_{I_{i}^{*}}\left(x(t)-\pi_{r}\left(t ; x ; I_{i}^{*}\right)\right)^{p} d t \\
& +\sum_{i=2}^{n} \int_{I_{i}^{*}}\left(\sum_{j=2}^{i}\left(\pi_{r}\left(t ; x ; I_{j}^{*}\right)-\pi_{r}\left(t ; x ; I_{j-1}^{*}\right)\right)\right)^{p} d t .
\end{aligned}
$$


Из определения (3.10) многочленов $\pi_{r}\left(\cdot ; x ; I_{i}^{*}\right)$ следует, что при $j=2, \ldots, i$, где $i=2, \ldots, n$, справедливы равенства

$$
\begin{gathered}
\pi_{r}\left(t ; x ; I_{j}^{*}\right)-\pi_{r}\left(t ; x ; I_{j-1}^{*}\right)=\sum_{k=0}^{r-1}\left(x^{(k)}\left(t_{j-1}^{*}\right)-\pi_{r}^{(k)}\left(t_{j-1}^{*} ; x ; I_{j-1}^{*}\right)\right) \frac{\left(t-t_{j-1}^{*}\right)^{k}}{k !} \\
=\sum_{k=0}^{r-1}\left(x^{(k)}\left(t_{j-1}^{*}\right)-\pi_{r-k}\left(t_{j-1}^{*} ; x^{(k)} ; I_{j-1}^{*}\right)\right) \frac{\left(t-t_{j-1}^{*}\right)^{k}}{k !}
\end{gathered}
$$

где

$$
\pi_{r-k}\left(t_{j-1}^{*} ; x^{(k)} ; I_{j-1}^{*}\right):=\sum_{l=0}^{r-1-k} \frac{x^{(k+l)}\left(t_{j-2}^{*}\right)}{l !}\left(t_{j-1}^{*}-t_{j-2}^{*}\right)^{l} .
$$

Если $0 \leqslant k<r-1$, то, воспользовавшись формулой Тейлора и учитьвая монотонность производной $x^{(r-1)}$, получаем

$$
\begin{aligned}
& x^{(k)}\left(t_{j-1}^{*}\right)-\pi_{r-k}\left(t_{j-1}^{*} ; x^{(k)} ; I_{j-1}^{*}\right) \\
& \quad=\frac{1}{(r-k-2) !} \int_{I_{j-1}^{*}}\left(x^{(r-1)}(\tau)-x^{(r-1)}\left(t_{j-2}^{*}\right)\right)\left(t_{j-1}^{*}-\tau\right)^{r-k-2} d \tau \\
& \quad \geqslant \frac{\left|I_{j-1}^{*}\right|^{-k}}{(r-k-2) !} \int_{I_{j-1}^{*}}\left(x^{(r-1)}(\tau)-x^{(r-1)}\left(t_{j-2}^{*}\right)\right)\left(t_{j-1}^{*}-\tau\right)^{r-2} d \tau .
\end{aligned}
$$

Если же $k=r-1$, то в силу монотонности производной $x^{(r-1)}$ имеем

$$
\begin{aligned}
& x^{(r-1)}\left(t_{j-1}^{*}\right)-\pi_{1}\left(t_{j-1}^{*} ; x^{(r-1)} ; I_{j-1}^{*}\right)=x^{(r-1)}\left(t_{j-1}^{*}\right)-x^{(r-1)}\left(t_{j-2}^{*}\right) \\
& \geqslant\left|I_{j-1}^{*}\right|^{-r+1} \int_{I_{j-1}^{*}}\left(x^{(r-1)}(\tau)-x^{(r-1)}\left(t_{j-2}^{*}\right)\right)\left(t_{j-1}^{*}-\tau\right)^{r-2} d \tau .
\end{aligned}
$$

Но тогда из (3.21) и (3.22) следует, что

$$
\begin{aligned}
\sum_{k=0}^{r-1}\left(x^{(k)}\left(t_{j-1}^{*}\right)-\right. & \left.\pi_{r-k}\left(t_{j-1}^{*} ; x^{(k)} ; I_{j-1}^{*}\right)\right) \frac{\left(t-t_{j-1}^{*}\right)^{k}}{k !} \\
\geqslant & \frac{1}{(r-1) !}\left(\sum_{k=0}^{r-1}\left(\left|I_{j-1}^{*}\right|^{-1}\left(t-t_{j-1}^{*}\right)\right)^{k}\right) \\
& \times \int_{I_{j-1}^{*}}\left(x^{(r-1)}(\tau)-x^{(r-1)}\left(t_{j-2}^{*}\right)\right)\left(t_{j-1}^{*}-\tau\right)^{r-2} d \tau .
\end{aligned}
$$

Учитывая, что $\left(\begin{array}{c}r-1 \\ k\end{array}\right) \leqslant 2^{r-1}, k=0, \ldots, r-1$, имеем

$$
\begin{aligned}
\sum_{k=0}^{r-1}\left(\left|I_{j-1}^{*}\right|^{-1}\left(t-t_{j-1}^{*}\right)\right)^{k} & \geqslant \frac{1}{2^{r-1}}\left(\sum_{k=0}^{r-1}\left(\begin{array}{c}
r-1 \\
k
\end{array}\right)\left(\left|I_{j-1}^{*}\right|^{-1}\left(t-t_{j-1}^{*}\right)\right)^{k}\right) \\
& =\frac{\left|I_{j-1}^{*}\right|^{-r+1}}{2^{r-1}}\left(\left|I_{j-1}^{*}\right|+\left(t-t_{j-1}^{*}\right)\right)^{r-1} .
\end{aligned}
$$


Полагая $\bar{t}_{i}^{*}:=\left(t_{i-1}^{*}+t_{i}^{*}\right) / 2$, отмечаем, что при $j=2, \ldots, i$, где $i=2, \ldots, n$, будут вьполняться неравенства

$$
\left|I_{j-1}^{*}\right|+\left(t-t_{j-1}^{*}\right) \geqslant \frac{1}{2} \sum_{k=j-1}^{i}\left|I_{k}^{*}\right|, \quad t \in\left[\bar{t}_{i}^{*}, t_{i}^{*}\right], \quad i=2, \ldots, n
$$

Ясно также, что для $t \in I_{j-1}^{*}, j=2, \ldots, n$, в силу монотонности производной $x^{(r-1)}$ выполняются неравенства

$$
\begin{aligned}
\int_{I_{j-1}^{*}}\left(x^{(r-1)}(\tau)-x^{(r-1)}\left(t_{j-2}^{*}\right)\right)\left(t_{j-1}^{*}-\tau\right)^{r-2} d \tau \\
\quad \geqslant \int_{t_{j-2}^{*}}^{t}\left(x^{(r-1)}(\tau)-x^{(r-1)}\left(t_{j-2}^{*}\right)\right)(t-\tau)^{r-2} d \tau
\end{aligned}
$$

из которых следует, что

$$
\int_{I_{j-1}^{*}}\left(x^{(r-1)}(\tau)-x^{(r-1)}\left(t_{j-2}^{*}\right)\right)\left(t_{j-1}^{*}-\tau\right)^{r-2} d \tau \geqslant\left|I_{j-1}^{*}\right|^{-1 / q} \mu_{r, q}\left(\bar{I}_{j-1}^{*} ; x\right) .
$$

Но тогда из (3.20) и (3.23)-(3.26) следует, что при $j=2, \ldots, i$, где $i=2, \ldots, n$, для $t \in\left[\bar{t}_{i}^{*}, t_{i}^{*}\right]$ будут выполняться неравенства

$$
\pi_{r}\left(t ; x ; I_{j}^{*}\right)-\pi_{r}\left(t ; x ; I_{j-1}^{*}\right) \geqslant \frac{\left|I_{j-1}^{*}\right|^{-r+1-1 / q}}{2^{2 r-2}(r-1) !}\left(\sum_{k=j-1}^{i}\left|I_{k}^{*}\right|\right)^{r-1} \mu_{r, q}\left(\bar{I}_{j-1}^{*} ; x\right) .
$$

Поэтому при $i=2, \ldots, n$ будут справедливы неравенства

$$
\begin{aligned}
\int_{I_{i}^{*}}\left(\sum_{j=2}^{i}(\right. & \left.\left.\pi_{r}\left(t ; x ; I_{j}^{*}\right)-\pi_{r}\left(t ; x ; I_{j-1}^{*}\right)\right)\right)^{p} d t \\
\geqslant & \int_{\bar{t}_{i}^{*}}^{t_{i}^{*}}\left(\sum_{j=2}^{i}\left(\pi_{r}\left(t ; x ; I_{j}^{*}\right)-\pi_{r}\left(t ; x ; I_{j-1}^{*}\right)\right)\right)^{p} d t \\
\geqslant & \frac{\left|I_{i}^{*}\right|}{2^{(2 r-2) p+1}((r-1) !) p}\left(\min _{2 \leqslant j \leqslant i} \mu_{r, q}\left(\bar{I}_{j-1}^{*} ; x\right)\right)^{p} \\
& \quad \times\left(\sum_{j=2}^{i}\left|I_{j-1}^{*}\right|^{-r+1-1 / q}\left(\sum_{k=j-1}^{i}\left|I_{k}^{*}\right|\right)^{r-1}\right)^{p} .
\end{aligned}
$$

Наряду с этим при $i=1, \ldots, n$ из формулы Тейлора и неравенства Гёльдера следует, что

$$
\int_{I_{i}^{*}}\left(x(t)-\pi_{r}\left(t ; x ; I_{i}^{*}\right)\right)^{p} d t \geqslant \frac{\left|I_{i}^{*}\right|^{1-p / q}}{((r-2) !)^{p}}\left(\mu_{r, q}\left(\bar{I}_{i}^{*} ; x\right)\right)^{p} .
$$


Подставляя оценки (3.27) и (3.28) в (3.19), имеем

$$
\begin{aligned}
\|x\|_{L_{p}\left(\bar{I}_{+}\right)}^{p} \geqslant & c\left(\sum_{i=1}^{n}\left|I_{i}^{*}\right|^{1-p / q}+\sum_{i=2}^{n}\left|I_{i}^{*}\right|\left(\sum_{j=2}^{i}\left|I_{j-1}^{*}\right|^{-r+1-1 / q}\left(\sum_{k=j-1}^{i}\left|I_{k}^{*}\right|\right)^{r-1}\right)^{p}\right) \\
& \times\left(\min _{1 \leqslant i \leqslant n} \mu_{r, q}\left(\bar{I}_{i}^{*} ; x\right)\right)^{p},
\end{aligned}
$$

где $c=2^{-2 r p+2 p-1}((r-1) !)^{-p}$. Воспользовавшись неравенством $\left(|a|^{p}+|b|^{p}\right)^{1 / p} \geqslant$ $2^{1 / p-1}(|a|+|b|)$, получим

$$
\begin{gathered}
\left(\sum_{i=1}^{n}\left|I_{i}^{*}\right|^{1-p / q}+\sum_{i=2}^{n}\left|I_{i}^{*}\right|\left(\sum_{j=2}^{i}\left|I_{j-1}^{*}\right|^{-r+1-1 / q}\left(\sum_{k=j-1}^{i}\left|I_{k}^{*}\right|\right)^{r-1}\right)^{p}\right)^{1 / p} \\
\geqslant 2^{1 / p-1}\left(\sum_{i=1}^{n}\left(\left|I_{i}^{*}\right|^{1 / p} \sum_{j=1}^{i}\left|I_{j}^{*}\right|^{-r+1-1 / q}\left(\sum_{k=j}^{i}\left|I_{k}^{*}\right|\right)^{r-1}\right)^{p}\right)^{1 / p} .
\end{gathered}
$$

Следовательно,

$$
\begin{aligned}
& \min _{1 \leqslant i \leqslant n} \mu_{r, q}\left(\bar{I}_{i}^{*} ; x\right) \\
& \quad \leqslant c_{*}\|x\|_{L_{p}\left(\bar{I}_{+}\right)}\left(\sum_{i=1}^{n}\left(\left|I_{i}^{*}\right|^{1 / p} \sum_{j=1}^{i}\left|I_{j}^{*}\right|^{-r+1-1 / q}\left(\sum_{k=j}^{i}\left|I_{k}^{*}\right|\right)^{r-1}\right)^{p}\right)^{-1 / p}
\end{aligned}
$$

где $c_{*}=2^{2 r-1}(r-1)$ !. Нетрудно убедиться, что оценка (3.29) верна и при $p=\infty$.

Но тогда при $r>1$ и $1 \leqslant q<p \leqslant \infty$ из (3.18), (3.9) и (3.29) следует оценка

$$
\begin{aligned}
& \left\|x(\cdot)-\sigma_{r, n}\left(\cdot ; x ; \bar{I}_{+}\right)\right\|_{L_{q}\left(\bar{I}_{+}\right)} \\
& \quad \leqslant c^{*} n^{1 / q}\|x\|_{L_{p}\left(\bar{I}_{+}\right)}\left(\sum_{i=1}^{n}\left(\left|I_{i}^{*}\right|^{1 / p} \sum_{j=1}^{i}\left|I_{j}^{*}\right|^{-r+1-1 / q}\left(\sum_{k=j}^{i}\left|I_{k}^{*}\right|\right)^{r-1}\right)^{p}\right)^{-1 / p}
\end{aligned}
$$

где $c^{*}=2^{2 r-1}(r-1)$. Учитывая неравенство (3.16), видим, что оценка (3.30) верна и в случае $r=1$ с постоянной $c^{*}=2^{1-1 / p}$.

Теперь можем воспользоваться леммой 5 . Полагая в ней $\alpha:=r-1+1 / q$, $\beta:=r-1$ и $\delta_{i}:=\left|I_{i}^{*}\right|, i=1, \ldots, n$, получаем

$$
\left(\sum_{i=1}^{n}\left(\left|I_{i}^{*}\right|^{1 / p} \sum_{j=1}^{i}\left|I_{j}^{*}\right|^{-r+1-1 / q}\left(\sum_{k=j}^{i}\left|I_{k}^{*}\right|\right)^{r-1}\right)^{p}\right)^{-1 / p} \leqslant c n^{-r-1 / q}
$$

где $c=c(r, p, q)$.

Подставляя эту оценку в (3.30), имеем

$\left\|x(\cdot)-\sigma_{r, n}\left(\cdot ; x ; \bar{I}_{+}\right)\right\|_{L_{q}\left(\bar{I}_{+}\right)} \leqslant c\|x\|_{L_{p}\left(\bar{I}_{+}\right)} n^{-r}, \quad n>1, \quad 1 \leqslant q<p \leqslant \infty$, 
где $c=c(r, p, q)$. Напомним, что оценка (3.31) доказана при условии (3.9). Но, учитывая соотношения (3.3) и (3.8), видим, что оценка (3.31) верна и для $n=1$, а также для $n>1$ при условии (3.6).

Отметим теперь, что функция $y(t):=(-1)^{s} x(-t), t \in \bar{I}_{+}$, имеет на $\bar{I}_{+}$неотрицательные производные $y^{(k)}, k=0, \ldots, s-1$, и что $y^{(k)}(0)=0, k=0, \ldots, s-1$. Определив для функции $y$ сплайны $\sigma_{r, n}\left(\cdot ; y ; \bar{I}_{+}\right)$на отрезке $\bar{I}_{+}$аналогично тому, как определялись сплайны $\sigma_{r, n}\left(t ; x ; \bar{I}_{+}\right)$для функции $x$, полагаем

$$
\sigma_{r, n}\left(t ; x ; \bar{I}_{-}\right):=(-1)^{s} \sigma_{r, n}\left(-t ; y ; \bar{I}_{+}\right), \quad t \in \bar{I}_{-} .
$$

Ясно, что из (3.31) следует оценка

$$
\left\|x(\cdot)-\sigma_{r, n}\left(\cdot ; x ; \bar{I}_{-}\right)\right\|_{L_{q}\left(\bar{I}_{-}\right)} \leqslant c\|x\|_{L_{p}\left(\bar{I}_{-}\right)} n^{-r}, \quad n \geqslant 1, \quad 1 \leqslant q<p \leqslant \infty
$$

где $c=c(r, p, q)$.

Затем определяем сплайн $\sigma_{r, n}(\cdot ; x ; \bar{I})$ на всем отрезке $\bar{I}$, полагая

$$
\sigma_{r, n}(t ; x ; \bar{I}):= \begin{cases}\sigma_{r, n}\left(t ; x ; \bar{I}_{+}\right), & t \in \bar{I}_{+} \\ \sigma_{r, n}\left(t ; x ; \bar{I}_{-}\right), & t \in \bar{I}_{-}\end{cases}
$$

Тогда из (3.31) и (3.32) будет следовать, что для $r \leqslant s$ имеет место оценка

$$
\left\|x(\cdot)-\sigma_{r, n}(\cdot ; x ; \bar{I})\right\|_{L_{q}(\bar{I})} \leqslant c\|x\|_{L_{p}(\bar{I})} n^{-r}, \quad n \geqslant 1, \quad 1 \leqslant q<p \leqslant \infty,
$$

где $c=c(r, p, q)$.

Учитьвая, что при $r=s$ приближающие сплайны $\sigma_{s, n}(\cdot ; x ; \bar{I})$ уже построены, определяем приближаюшие сплайны в случае $r>s$, полагая

$$
\sigma_{r, n}(t ; x ; \bar{I}):=\sigma_{s, n}(t ; x ; \bar{I}), \quad t \in \bar{I} .
$$

Тогда из (3.33) немедленно следует оценка

$$
\left\|x(\cdot)-\sigma_{r, n}(\cdot ; x ; \bar{I})\right\|_{L_{q}(\bar{I})} \leqslant c\|x\|_{L_{p}(\bar{I})} n^{-\min \{r, s\}}, \quad 1 \leqslant q<p \leqslant \infty,
$$

где $c=c(r, s, p, q)$. Кроме того, очевидно, что сплайны $\sigma_{r, n}(\cdot ; x ; \bar{I})$ имеют не более $2(n-1)$ свободных узлов на отрезке $\bar{I}$.

Отметим, что неравенства (3.35) установлены для $s$-монотонных функций из пространства $C^{s-1}(\bar{I})$ и при дополнительном условии (3.1). Чтобы получить оценки вида (3.35) в обшем случае, т.е. для функций $x \in \Delta_{+}^{s} L_{p}(I), 1 \leqslant p \leqslant \infty$, на интервале $I=(-1,1)$, необходимо построить более гладкие функции, сохранив при этом $s$-монотонность и не увеличив сушественно норму в $L_{p}$.

Для этого поступим следуюшим образом. Пусть $x \in \Delta_{+}^{s} L_{p}(I), 1 \leqslant p \leqslant \infty$. Полагая

$$
\widetilde{x}(t):=x(t)-\pi_{s}(t ; x), \quad t \in I,
$$

где

$$
\pi_{s}(t ; x):=\sum_{k=0}^{s-1} \frac{x^{(k)}(0)}{k !} t^{k}, \quad t \in I,
$$


и воспользовавшись леммой 3 , имеем

$$
\|\widetilde{x}\|_{L_{p}(I)} \leqslant \widetilde{c}\|x\|_{L_{p}(I)},
$$

где $\widetilde{c}=\widetilde{c}(s, p)$.

Ясно, что $\widetilde{x}$ является $s$-монотонной функцией на $I$. Кроме того, нетрудно убедиться, что для всех $k=0, \ldots, s-1$ выполняются неравенства $\widetilde{x}^{(k)}(t) \geqslant 0$, $t \in I_{+}:=[0,1)$. Если же $t \in I_{-}:=(-1,0]$, то $(-1)^{s-k} \widetilde{x}^{(k)}(t) \geqslant 0, k=0, \ldots, s-1$. Отметим также, что все производные $\widetilde{x}^{(k)}, k=0, \ldots, s-1$, являются неубывающими на $I_{+}$функциями, а на $I_{-}$происходит чередование монотонности для производных $\widetilde{x}^{(k)}, k=0, \ldots, s-1$.

Пусть $\epsilon \in(0,1 / 2)$, а $t_{\epsilon}^{-}:=-1+\epsilon$ и $t_{\epsilon}^{+}:=1-\epsilon$. Полагая

$$
\begin{aligned}
& \pi_{s}\left(t ; \widetilde{x} ; t_{\epsilon}^{-}\right):=\sum_{k=0}^{s-1} \frac{\widetilde{x}^{(k)}\left(t_{\epsilon}^{-}\right)}{k !}\left(t-t_{\epsilon}^{-}\right)^{k}, \quad t \in \mathbb{R}, \\
& \pi_{s}\left(t ; \widetilde{x} ; t_{\epsilon}^{+}\right):=\sum_{k=0}^{s-1} \frac{\widetilde{x}^{(k)}\left(t_{\epsilon}^{+}\right)}{k !}\left(t-t_{\epsilon}^{+}\right)^{k}, \quad t \in \mathbb{R},
\end{aligned}
$$

определяем на $\mathbb{R}$ следующие функции:

$$
\widetilde{x}_{\epsilon}(t):= \begin{cases}\pi_{s}\left(t ; \widetilde{x} ; t_{\epsilon}^{-}\right), & t \in\left(-\infty, t_{\epsilon}^{-}\right], \\ \widetilde{x}(t), & t \in\left(t_{\epsilon}^{-}, t_{\epsilon}^{+}\right), \\ \pi_{s}\left(t ; \widetilde{x} ; t_{\epsilon}^{+}\right), & t \in\left[t_{\epsilon}^{+},+\infty\right) .\end{cases}
$$

Ясно, что функции $\widetilde{x}_{\epsilon}$ Являются $s$-монотонньми на любом конечном интервале и при этом $0 \leqslant(-1)^{s} \pi_{s}\left(t ; \widetilde{x} ; t_{\epsilon}^{-}\right) \leqslant(-1)^{s} \widetilde{x}(t)$ для $t \in\left(-1, t_{\epsilon}^{-}\right)$, а для $t \in\left(t_{\epsilon}^{+}, 1\right)$ вьполняются неравенства $0 \leqslant \pi_{s}\left(t ; \widetilde{x} ; t_{\epsilon}^{-}\right) \leqslant \widetilde{x}(t)$. Очевидно также, что

$$
\left\|\widetilde{x}_{\epsilon}\right\|_{L_{p}(I)} \leqslant\|\widetilde{x}\|_{L_{p}(I)}
$$

Кроме того, из определения функции $\widetilde{x}_{\epsilon}$ и неравенства Гёльдера следует, что

$$
\begin{aligned}
& \left\|\widetilde{x}-\widetilde{x}_{\epsilon}\right\|_{L_{q}(I)} \leqslant\left\|\widetilde{x}(\cdot)-\pi_{s}\left(\cdot ; \widetilde{x} ; t_{\epsilon}^{-}\right)\right\|_{L_{q}\left(-1, t_{\epsilon}^{-}\right)}+\left\|\widetilde{x}(\cdot)-\pi_{s}\left(\cdot ; \widetilde{x} ; t_{\epsilon}^{+}\right)\right\|_{L_{q}\left(t_{\epsilon}^{+}, 1\right)} \\
& \quad \leqslant\|\widetilde{x}\|_{L_{q}\left(-1, t_{\epsilon}^{-}\right)}+\|\widetilde{x}\|_{L_{q}\left(t_{\epsilon}^{+}, 1\right)} \leqslant \epsilon^{1 / q-1 / p}\left(\|\widetilde{x}\|_{L_{p}\left(-1, t_{\epsilon}^{-}\right)}+\|\widetilde{x}\|_{L_{p}\left(t_{\epsilon}^{+}, 1\right)}\right) \\
& \quad \leqslant 2 \epsilon^{1 / q-1 / p}\|\widetilde{x}\|_{L_{p}(I)} .
\end{aligned}
$$

Таким образом, если $1 \leqslant q<p \leqslant \infty$, то

$$
\left\|\widetilde{x}-\widetilde{x}_{\epsilon}\right\|_{L_{q}(I)} \rightarrow 0, \quad \epsilon \rightarrow 0 .
$$

Наряду с этим из леммы 3 следует, что на интервалах $J_{\epsilon}^{-}:=(-1,-1+2 \epsilon)$ и $J_{\epsilon}^{+}:=(1-2 \epsilon, 1)$ выполняются соответственно неравенства

$$
\begin{aligned}
& \left\|\pi_{s}\left(\cdot ; \widetilde{x} ; t_{\epsilon}^{-}\right)\right\|_{L_{p}\left(J_{\epsilon}^{-}\right)} \leqslant c_{\epsilon}\|\widetilde{x}\|_{L_{p}\left(J_{\epsilon}^{-}\right)}, \\
& \left\|\pi_{s}\left(\cdot ; \widetilde{x} ; t_{\epsilon}^{+}\right)\right\|_{L_{p}\left(J_{\epsilon}^{+}\right)} \leqslant c_{\epsilon}\|\widetilde{x}\|_{L_{p}\left(J_{\epsilon}^{+}\right)},
\end{aligned}
$$

где $c_{\epsilon}=c_{\epsilon}(s, p)$. 
Кроме того, из леммы 7 следует, что на интервалах $J_{2 \epsilon}^{-}:=(-1-\epsilon,-1+3 \epsilon)$ и $J_{2 \epsilon}^{+}:=(1-3 \epsilon, 1+\epsilon)$ выполняются соответственно неравенства

$$
\begin{aligned}
\left\|\pi_{s}\left(\cdot ; \widetilde{x} ; t_{\epsilon}^{-}\right)\right\|_{L_{p}\left(J_{2 \epsilon}^{-}\right)} & \leqslant \widehat{c}_{\epsilon}\left\|\pi_{s}\left(\cdot ; \widetilde{x} ; t_{\epsilon}^{-}\right)\right\|_{L_{p}\left(J_{\epsilon}^{-}\right)}, \\
\left\|\pi_{s}\left(\cdot ; \widetilde{x} ; t_{\epsilon}^{+}\right)\right\|_{L_{p}\left(J_{2 \epsilon}^{+}\right)} & \leqslant \widehat{c}_{\epsilon}\left\|\pi_{s}\left(\cdot ; \widetilde{x} ; t_{\epsilon}^{+}\right)\right\|_{L_{p}\left(J_{\epsilon}^{+}\right)},
\end{aligned}
$$

где $\widehat{c}_{\epsilon}=\widehat{c}_{\epsilon}(s, p)$.

Но тогда из (3.37), (3.38) и (3.40)-(3.43) следует, что на интервале $I_{\epsilon}:=(-1-\epsilon$, $1+\epsilon)$ будет выполняться неравенство

$$
\left\|\widetilde{x}_{\epsilon}\right\|_{L_{p}\left(I_{\epsilon}\right)} \leqslant \widetilde{c}_{\epsilon}\|x\|_{L_{p}(I)}
$$

где $\widetilde{c}_{\epsilon}=\widetilde{c}_{\epsilon}(s, p)$.

Теперь сгладим функции $\widetilde{x}_{\epsilon}$. При каждом $\varepsilon \in(0, \epsilon)$ обозначим через

$$
\widetilde{x}_{\epsilon, \varepsilon}(t):=\frac{1}{\varepsilon} \int_{-\varepsilon / 2}^{\varepsilon / 2} \widetilde{x}_{\epsilon}(t+\tau) d \tau, \quad t \in \mathbb{R},
$$

$\varepsilon$-усреднение по Стеклову функций $\widetilde{x}_{\epsilon}$. Ясно, что функции $\widetilde{x}_{\epsilon, \varepsilon}$ ЯВляются $s$-монотонными на отрезке $\bar{I}=[-1,1]$ и имеют на $\bar{I}$ непрерывную производную порядка $s-1$. Кроме того, из обобшенного неравенства Минковского для интегралов и непрерывности в целом функций из $L_{q}, 1 \leqslant q<\infty$, следует, что

$$
\left\|\widetilde{x}_{\epsilon}-\widetilde{x}_{\epsilon, \varepsilon}\right\|_{L_{q}(\bar{I})} \rightarrow 0, \quad \varepsilon \rightarrow 0 .
$$

Очевидно также, что

$$
\left\|\widetilde{x}_{\epsilon, \varepsilon}\right\|_{L_{p}(I)} \leqslant\left\|\widetilde{x}_{\epsilon}\right\|_{L_{p}\left(\bar{I}_{\epsilon}\right)}
$$

Полагая теперь

$$
\pi_{s}\left(t ; \widetilde{x}_{\epsilon, \varepsilon}\right):=\sum_{k=0}^{s-1} \frac{\widetilde{x}_{\epsilon, \varepsilon}^{(k)}(0)}{k !} t^{k}, \quad t \in \bar{I},
$$

рассматриваем функции

$$
\breve{x}_{\epsilon, \varepsilon}(t):=\widetilde{x}_{\epsilon, \varepsilon}(t)-\pi_{s}\left(t ; \widetilde{x}_{\epsilon, \varepsilon}\right), \quad t \in \bar{I} .
$$

Из леммы 3 следует, что

$$
\left\|\breve{x}_{\epsilon, \varepsilon}\right\|_{L_{p}(\bar{I})} \leqslant \breve{c}_{\epsilon, \varepsilon}\left\|\widetilde{x}_{\epsilon, \varepsilon}\right\|_{L_{p}(\bar{I})}
$$

где $\breve{c}_{\epsilon, \varepsilon}=\breve{c}_{\epsilon, \varepsilon}(s, p)$. При этом $\breve{x}_{\epsilon, \varepsilon} \in \Delta_{+}^{s} C^{s-1}(\bar{I})$ и $\breve{x}_{\epsilon, \varepsilon}^{(k)}(0)=0, k=0, \ldots, s-1$.

Таким образом, функции $\breve{x}_{\epsilon, \varepsilon}$ удовлетворяют всем условиям, при которых установлены неравенства вида (3.35). Поэтому, определив для функций $\breve{x}_{\epsilon, \varepsilon}$ сплайны $\sigma_{r, n}\left(\cdot ; \breve{x}_{\epsilon, \varepsilon} ; \bar{I}\right)$ согласно $(3.2),(3.7),(3.11)$ и $(3.34)$, имеем

$$
\left\|\breve{x}_{\epsilon, \varepsilon}(\cdot)-\sigma_{r, n}\left(\cdot ; \breve{x}_{\epsilon, \varepsilon} ; \bar{I}\right)\right\|_{L_{q}(\bar{I})} \leqslant c_{\epsilon, \varepsilon}\left\|\breve{x}_{\epsilon, \varepsilon}\right\|_{L_{p}(\bar{I})} n^{-\min \{r, s\}},
$$

где $c_{\epsilon, \varepsilon}=c_{\epsilon, \varepsilon}(r, s, p, q)$. 
Теперь воспользуемся этими сплайнами для построения сплайнов, приближаюших функции $x \in \Delta_{+}^{s} L_{p}(I)$.

Если $r \geqslant s$, то для каждой функции $x \in \Delta_{+}^{s} L_{p}(I)$ полагаем

$$
\sigma_{r, s, n}(t ; x ; I):=\sigma_{r, n}\left(t ; \breve{x}_{\epsilon, \varepsilon} ; \bar{I}\right)+\pi_{s}\left(t ; \widetilde{x}_{\epsilon, \varepsilon}\right)+\pi_{s}(t ; x), \quad t \in I,
$$

где $\pi_{s}\left(\cdot ; \widetilde{x}_{\epsilon, \varepsilon}\right)$ и $\pi_{s}(\cdot ; x)$ - многочлены из $(3.47)$ и $(3.36)$ соответственно.

Отметим, что из определения сплайнов $\sigma_{r, n}\left(\cdot ; \breve{x}_{\epsilon, \varepsilon} ; \bar{I}\right)$ следуют тождества

$$
\begin{aligned}
\sigma_{r, n}\left(t ; \pi_{s}\left(\cdot ; \widetilde{x}_{\epsilon, \varepsilon}\right) ; \bar{I}\right) & =\pi_{s}\left(t ; \widetilde{x}_{\epsilon, \varepsilon}\right), & & t \in \bar{I} \\
\sigma_{r, n}\left(t ; \pi_{s}(\cdot ; x) ; \bar{I}\right) & =\pi_{s}(t ; x), & & t \in \bar{I} .
\end{aligned}
$$

Поэтому, учитывая также тождество

$$
x(t)=\breve{x}_{\epsilon, \varepsilon}(t)+\left(\widetilde{x}_{\epsilon}(t)-\widetilde{x}_{\epsilon, \varepsilon}(t)\right)+\left(\widetilde{x}(t)-\widetilde{x}_{\epsilon}(t)\right)+\pi_{s}\left(t ; \widetilde{x}_{\epsilon, \varepsilon}\right)+\pi_{s}(t ; x),
$$

имеем

$x(t)-\sigma_{r, s, n}(t ; x ; I)=\left(\breve{x}_{\epsilon, \varepsilon}(t)-\sigma_{r, n}\left(t ; \breve{x}_{\epsilon, \varepsilon} ; \bar{I}\right)\right)+\left(\widetilde{x}_{\epsilon}(t)-\widetilde{x}_{\epsilon, \varepsilon}(t)\right)+\left(\widetilde{x}(t)-\widetilde{x}_{\epsilon}(t)\right)$.

Но тогда

$$
\begin{aligned}
& \left\|x(\cdot)-\sigma_{r, s, n}(\cdot ; x ; I)\right\|_{L_{q}(I)} \\
& \quad \leqslant\left\|\breve{x}_{\epsilon, \varepsilon}(\cdot)-\sigma_{r, n}\left(\cdot ; \breve{x}_{\epsilon, \varepsilon} ; \bar{I}\right)\right\|_{L_{q}(\bar{I})}+\left\|\widetilde{x}_{\epsilon}-\widetilde{x}_{\epsilon, \varepsilon}\right\|_{L_{q}(\bar{I})}+\left\|\widetilde{x}-\widetilde{x}_{\epsilon}\right\|_{L_{q}(\bar{I})} .
\end{aligned}
$$

Из этого неравенства, а также из (3.39), (3.45) и (3.49) следует, что для $r \geqslant s$ при достаточно малых $\epsilon$ и $\varepsilon$ будет иметь место оценка

$$
\left\|x(\cdot)-\sigma_{r, s, n}(\cdot ; x ; I)\right\|_{L_{q}(I)} \leqslant c\|x\|_{L_{p}(I)} n^{-s}, \quad n \geqslant 1, \quad 1 \leqslant q<p \leqslant \infty,
$$

где $c=c(r, s, p, q)$.

Если же $r<s$, то функция $\sigma_{r, s, n}(\cdot ; x ; I)$, определенная согласно $(3.50)$, не является сплайном порядка $\leqslant r$. Поэтому необходимо заменить многочлены $\pi_{s}\left(\cdot ; \widetilde{x}_{\epsilon, \varepsilon}\right)$ и $\pi_{s}(\cdot ; x)$ на сплайны порядка $\leqslant r$, которые достаточно хорошо приближают эти многочлены.

Воспользовавшись леммой 7 , имеем

$$
\left\|\pi_{s}^{(r)}\left(\cdot ; \widetilde{x}_{\epsilon, \varepsilon}\right)\right\|_{L_{\infty}(I)} \leqslant c\left\|\pi_{s}\left(\cdot ; \widetilde{x}_{\epsilon, \varepsilon}\right)\right\|_{L_{p}(I)},
$$

где $c=c(r, s, p)$. Но тогда из $(3.37),(3.44),(3.46),(3.48)$ и (3.53) следует, что

$$
\left\|\pi_{s}^{(r)}\left(\cdot ; \widetilde{x}_{\epsilon, \varepsilon}\right)\right\|_{L_{\infty}(I)} \leqslant c^{\prime}\|x\|_{L_{p}(I)},
$$

где $c^{\prime}=c^{\prime}(r, s, p)$. Аналогично, из (3.37) и леммы 7 следует, что

$$
\left\|\pi_{s}^{(r)}(\cdot ; x)\right\|_{L_{\infty}(I)} \leqslant c^{\prime \prime}\|x\|_{L_{p}(I)}
$$

где $c^{\prime \prime}=c^{\prime \prime}(r, s, p)$. 
Воспользовавшись неравенствами (3.54) и (3.55), а также известными оценками приближения сплайнами функций из пространств Соболева (см., например, [18; $\S 3.1$, теорема 1$])$, найдем сплайны $\varsigma_{r, n}\left(\cdot ; \pi_{s}\left(\cdot ; \widetilde{x}_{\epsilon, \varepsilon}\right) ; I\right)$ и $\varsigma_{r, n}\left(\cdot ; \pi_{s}(\cdot ; x) ; I\right)$ порядка $\leqslant r$ с не более чем $n-1$ свободньми узлами на $I$ такие, что

$$
\begin{aligned}
\left\|\pi_{s}\left(\cdot ; \widetilde{x}_{\epsilon, \varepsilon}\right)-\varsigma_{r, n}\left(\cdot ; \pi_{s}\left(\cdot ; \widetilde{x}_{\epsilon, \varepsilon}\right) ; I\right)\right\|_{L_{q}(I)} \leqslant \bar{c}\|x\|_{L_{p}(I)} n^{-r}, & n \geqslant 1 \\
\left\|\pi_{s}(\cdot ; x)-\varsigma_{r, n}\left(\cdot ; \pi_{s}(\cdot ; x) ; I\right)\right\|_{L_{q}(I)} \leqslant \bar{c}\|x\|_{L_{p}(I)} n^{-r}, & n \geqslant 1
\end{aligned}
$$

где $\bar{c}=\bar{c}(r, s, p, q)$.

Теперь можем определить приближаюшие сплайны порядка $\leqslant r$ и в случае $r<s$. В этом случае полагаем

$$
\sigma_{r, s, n}(t ; x ; I):=\sigma_{r, n}\left(t ; \breve{x}_{\epsilon, \varepsilon} ; \bar{I}\right)+\varsigma_{r, n}\left(t ; \pi_{s}\left(\cdot ; \widetilde{x}_{\epsilon, \varepsilon}\right) ; I\right)+\varsigma_{r, n}\left(t ; \pi_{s}(\cdot ; x) ; I\right),
$$

где сплайны $\sigma_{r, n}\left(\cdot ; \breve{x}_{\epsilon, \varepsilon} ; \bar{I}\right), \varsigma_{r, n}\left(\cdot ; \pi_{s}\left(\cdot ; \widetilde{x}_{\epsilon, \varepsilon}\right) ; I\right)$ и $\varsigma_{r, n}\left(\cdot ; \pi_{s}(\cdot ; x) ; I\right)$ удовлетворяют неравенствам (3.49), (3.56) и (3.57) соответственно.

Очевидно, что из (3.51) и (3.58) следует тождество

$$
\begin{aligned}
& x(t)- \sigma_{r, s, n}(t ; x ; I)=\left(\breve{x}_{\epsilon, \varepsilon}(t)-\sigma_{r, n}\left(t ; \breve{x}_{\epsilon, \varepsilon} ; \bar{I}\right)\right)+\left(\widetilde{x}_{\epsilon}(t)-\widetilde{x}_{\epsilon, \varepsilon}(t)\right)+\left(\widetilde{x}(t)-\widetilde{x}_{\epsilon}(t)\right) \\
&+\left(\pi_{s}\left(t ; \widetilde{x}_{\epsilon, \varepsilon}\right)-\varsigma_{r, n}\left(t ; \pi_{s}\left(\cdot ; \widetilde{x}_{\epsilon, \varepsilon}\right) ; I\right)\right)+\left(\pi_{s}(t ; x)-\varsigma_{r, n}\left(t ; \pi_{s}(\cdot ; x) ; I\right)\right) .
\end{aligned}
$$

Но тогда

$$
\begin{aligned}
\| x(\cdot)- & \sigma_{r, s, n}(\cdot ; x ; I)\left\|_{L_{q}(I)} \leqslant\right\| \breve{x}_{\epsilon, \varepsilon}(\cdot)-\sigma_{r, n}\left(\cdot ; \breve{x}_{\epsilon, \varepsilon} ; \bar{I}\right)\left\|_{L_{q}(I)}+\right\| \widetilde{x}_{\epsilon}-\widetilde{x}_{\epsilon, \varepsilon} \|_{L_{q}(I)} \\
& +\left\|\widetilde{x}-\widetilde{x}_{\epsilon}\right\|_{L_{q}(I)}+\left\|\pi_{s}\left(\cdot ; \widetilde{x}_{\epsilon, \varepsilon}\right)-\varsigma_{r, n}\left(\cdot ; \pi_{s}\left(\cdot ; \widetilde{x}_{\epsilon, \varepsilon}\right) ; I\right)\right\|_{L_{q}(I)} \\
& +\left\|\pi_{s}(\cdot ; x)-\varsigma_{r, n}\left(\cdot ; \pi_{s}(\cdot ; x) ; I\right)\right\|_{L_{q}(I)} .
\end{aligned}
$$

Из этого неравенства, а также соотношений (3.39), (3.45), (3.49), (3.56) и (3.57) видно, что при $r<s$ для достаточно малых $\epsilon$ и $\varepsilon$ будет справедлива оценка

$$
\left\|x(\cdot)-\sigma_{r, s, n}(\cdot ; x ; I)\right\|_{L_{q}(I)} \leqslant c\|x\|_{L_{p}(I)} n^{-r}, \quad n \geqslant 1, \quad 1 \leqslant q<p \leqslant \infty
$$

где $c=c(r, s, p, q)$.

Объединяя оценки (3.52) и (3.59), имеем

$$
\left\|x(\cdot)-\sigma_{r, s, n}(\cdot ; x ; I)\right\|_{L_{q}(I)} \leqslant c\|x\|_{L_{p}(I)} n^{-\min \{r, s\}}, \quad 1 \leqslant q<p \leqslant \infty,
$$

где $r, s \in \mathbb{N}$, а $c=c(r, s, p, q)$.

При этом ясно, что сплайны $\sigma_{r, s, n}(\cdot ; x ; I)$, определяемые согласно (3.50), (3.58), имеют на интервале $I=(-1,1)$ не более $3(n-1)$ свободных узлов. Поэтому из (3.60) следует, что

$$
E\left(\Delta_{+}^{s} B_{p}, \Sigma_{r, n}\right)_{L_{q}} \leqslant c n^{-\min \{r, s\}}, \quad n \geqslant 1, \quad 1 \leqslant q<p \leqslant \infty, \quad r, s \in \mathbb{N},
$$

где $c=c(r, s, p, q)$. Таким образом, оценки сверху доказаны и в общем случае.

ЗАмЕчАниЕ 9 . При доказательстве оценок сверху в теореме 1 осушествлялась “балансировка" отрезков $\bar{I}_{i}^{*}$. Отметим, что подобная идея "балансировки" применялась ранее в работах [7] и [12]. 
ЗАмЕчание 10. Сплайны, которые использовались для получения оценок сверху, являются, вообще говоря, разрывными. Нетрудно убедиться, что за счет некоторого увеличения числа узлов можно построить и более гладкие сплайны, даюшие тот же порядок приближения.

\section{§4. Доказательство оценок снизу в теореме 1}

Вначале рассмотрим случай $r \leqslant s$. Пусть на интервале $I:=(-1,1)$ задана функция

$$
x_{r}(t):=t^{r}, \quad t \in I,
$$

для которой включение $x_{r} \in \Delta_{+}^{s} B_{\infty}(I)$ является очевидньм.

Докажем, что

$$
E\left(x_{r}, \Sigma_{r, n}\right)_{L_{1}(I)} \geqslant 2^{-r+1} n^{-r}, \quad n \geqslant 1 .
$$

Для этого разобьем интервал $I$ произвольным образом на $n$ непересекающихся промежутков $I_{i}, i=1, \ldots, n$, таких, что $\bigcup_{i=1}^{n} I_{i}=I$. Обозначим через

$$
E\left(x_{r}, P_{r}\right)_{L_{1}\left(I_{i}\right)}:=\inf _{\pi_{r} \in P_{r}}\left\|x_{r}-\pi_{r}\right\|_{L_{1}\left(I_{i}\right)}, \quad i=1, \ldots, n,
$$

наилучшие приближения в $L_{1}\left(I_{i}\right)$ функции $x_{r}$ многочленами $\pi_{r} \in P_{r}\left(I_{i}\right)$.

Из определения многообразия $\Sigma_{r, n}(I)$ сплайнов следует, что

$$
E\left(x_{r}, \Sigma_{r, n}\right)_{L_{1}(I)}=\inf _{\left\{I_{i}\right\}_{1}^{n}} \sum_{i=1}^{n} E\left(x_{r}, P_{r}\right)_{L_{1}\left(I_{i}\right)},
$$

где inf взят по всем разбиениям $\left\{I_{i}\right\}_{1}^{n}$ интервала $I$ на промежутки $I_{i}$. А так как известно (см., например, $[18 ; \S 2.1 .4]$ ), что

$$
E\left(x_{r}, P_{r}\right)_{L_{1}\left(I_{i}\right)}=2^{-2 r}\left|I_{i}\right|^{r+1}, \quad i=1, \ldots, n,
$$

то, следовательно,

$$
\sum_{i=1}^{n} E\left(x_{r}, P_{r}\right)_{L_{1}\left(I_{i}\right)}=2^{-2 r} \sum_{i=1}^{n}\left|I_{i}\right|^{r+1} .
$$

Воспользовавшись затем известным неравенством

$$
\sum_{i=1}^{n}\left|a_{i}\right| \leqslant n^{1-1 /(r+1)}\left(\sum_{i=1}^{n}\left|a_{i}\right|^{r+1}\right)^{1 /(r+1)},
$$

получим

$$
\sum_{i=1}^{n}\left|I_{i}\right|^{r+1} \geqslant n^{-r}\left(\sum_{i=1}^{n}\left|I_{i}\right|\right)^{r+1}=2^{r+1} n^{-r} .
$$

Но тогда из (4.3) и (4.4) следует, что

$$
\sum_{i=1}^{n} E\left(x_{r}, P_{r}\right)_{L_{1}\left(I_{i}\right)} \geqslant 2^{-r+1} n^{-r} .
$$


Неравенство (4.1) - немедленное следствие соотношений (4.2) и (4.5). А в качестве простого следствия из неравенства (4.1) получаем оценку

$$
E\left(\Delta_{+}^{s} B_{p}, \Sigma_{r, n}\right)_{L_{q}} \geqslant c n^{-r}, \quad n \geqslant 1, \quad 1 \leqslant q<p \leqslant \infty, \quad r \leqslant s
$$

где $c=c(r, p, q)$. Итак, необходимая оценка снизу доказана для случая $r \leqslant s$.

Остается рассмотреть случай $r>s$. В этом случае построим при каждом $n \in \mathbb{N}$ функции из $\Delta_{+}^{s} B_{\infty}$, которые невозможно приблизить в метрике $L_{1}$ лучше, чем $c n^{-s}$, где $c=c(r, s)$.

Полагая

$$
\phi_{0}(t):=\left\{\begin{array}{l}
0, t \in(-\infty, 1] \\
1, \quad t \in(-1,1) \\
0, \quad t \in[1,+\infty)
\end{array}\right.
$$

определяем следуюшие функции:

$$
\phi_{s}(t):=\int_{-\infty}^{t}\left(\phi_{s-1}(2 \tau+1)-\phi_{s-1}(2 \tau-1)\right) d \tau, \quad t \in(-\infty,+\infty), \quad s \in \mathbb{N} .
$$

Ясно, что функции $\phi_{s}$ являются положительными на $(-1,1)$ и $\operatorname{supp} \phi_{s}=[-1,1]$.

При каждом $m \in \mathbb{N}$ разобьем интервал $I$ на $m$ промежутков $I_{i}, i=1, \ldots, m$, равной длины с концами соответственно в точках $t_{i-1}:=-1+2(i-1) / m$ и $t_{i}:=$ $-1+2 i / m$. Обозначая через $\bar{t}_{i}:=-1+(2 i-1) / m$ середины промежутков $I_{i}$, полагаем

$$
\phi_{s, m, i}(t):=\phi_{s}\left(m\left(t-\bar{t}_{i}\right)\right), \quad t \in I, \quad i=1, \ldots, m .
$$

Очевидно, что каждая из функций $\phi_{s, m, i}$ положительна на соответствуюшем интервале $\left(t_{i-1}, t_{i}\right)$, a supp $\phi_{s, m, i}=\left[t_{i-1}, t_{i}\right], i=1, \ldots, m$. Кроме того, нетрудно проверить, что при $s \in \mathbb{N}$ и всех $i=1, \ldots, m$ выполняются равенства

$$
\begin{gathered}
\left\|\phi_{s, m, i}\right\|_{L_{\infty}\left(I_{i}\right)}=2^{-s+1}, \quad\left\|\phi_{s, m, i}\right\|_{L_{1}\left(I_{i}\right)}=2^{-s+1} m^{-1}, \\
\left\|\phi_{s, m, i}^{(s)}\right\|_{L_{\infty}\left(I_{i}\right)}=2^{(s-1) s / 2} m^{s} .
\end{gathered}
$$

С помощью функций $\phi_{s, m, i}$ определим функции

$$
\varphi_{s, m}(t):=(-1)^{i} 2^{-(s-1) s / 2-1} m^{-s} \phi_{s, m, i}(t), \quad t \in I_{i}, \quad i=1, \ldots, m .
$$

Учитывая равенства (4.7), имеем

$$
\left\|\varphi_{s, m}\right\|_{L_{\infty}(I)}=2^{-s(s+1) / 2} m^{-s}, \quad\left\|\varphi_{s, m}^{(s)}\right\|_{L_{\infty}(I)}=2^{-1} .
$$

Полагая

$$
y_{s}(t):=2^{-1} t^{s}, \quad t \in I
$$

рассмотрим функции

$$
y_{s, m}(t):=y_{s}(t)+\varphi_{s, m}(t), \quad t \in I
$$


Воспользовавшись равенствами (4.9), легко убедиться, что $\left\|y_{s, m}\right\|_{L_{\infty}(I)} \leqslant 1$. При этом производная $y_{s, m}^{(s)}$ порядка $s$, существующая всюду на $I$ за исключением конечного числа точек, является кусочно постоянной неотрицательной функцией. Поэтому $y_{s, m} \in \Delta_{+}^{s} B_{\infty}(I)$ при всех $m \in \mathbb{N}$. Учитывая, что $y_{s}-$ алгебраический многочлен степени $s$, имеем

$$
E\left(y_{s, m}, \Sigma_{r, n}\right)_{L_{1}(I)} \geqslant E\left(\varphi_{s, m}, \Sigma_{r, n}\right)_{L_{1}(I)}, \quad n \geqslant 1, \quad m \in \mathbb{N}, \quad r>s .
$$

Докажем теперь, что при $m=8 r n$ будет справедлива оценка

$$
E\left(\varphi_{s, 8 r n}, \Sigma_{r, n}\right)_{L_{1}(I)} \geqslant c n^{-s}, \quad n \geqslant 1, \quad r>s
$$

где $c=c(r, s)$.

Для произвольного сплайна $\sigma_{r, n} \in \Sigma_{r, n}(I)$ обозначим через $I_{+}\left(\sigma_{r, n}\right)$ множество всех точек $t \in I$ таких, что $\sigma_{r, n}(t)>0$. Ясно, что число связных компонент множества $I_{+}\left(\sigma_{r, n}\right)$ не превышает $r n$. Все эти компоненты разобьем на две группы, обозначаемые через $G_{+}^{\prime}\left(\sigma_{r, n}\right)$ и $G_{+}^{\prime \prime}\left(\sigma_{r, n}\right)$. К группе $G_{+}^{\prime \prime}\left(\sigma_{r, n}\right)$ отнесем компоненты, каждая из которых пересекается более чем с одним из интервалов $I_{i}$ таких, что функция $\varphi_{s, 8 r n}$ является положительной на $I_{i}$. Остальные компоненты отнесем к группе $G_{+}^{\prime}\left(\sigma_{r, n}\right)$.

Допустим, что объединение всех компонент из группы $G_{+}^{\prime \prime}\left(\sigma_{r, n}\right)$ пересекается не более чем с $2 r n$ интервалами $I_{i}$, на которых функция $\varphi_{s}, 8 r n$ является положительной. Тогда все множество $I_{+}\left(\sigma_{r, n}\right)$ пересекается не более чем с $3 r n$ такими интервалами. Всего же имеется $4 r n$ интервалов $I_{i}$, на которых функция $\varphi_{s, 8 r n}$ положительна. Значит, на не менее чем $r n$ интервалах $I_{i}$ из $I$ функции $\sigma_{r, n}$ и $\varphi_{s, 8 r n}$ имеют разные знаки. Поэтому из (4.8) и (4.7) следует, что

$$
\left\|\varphi_{s, 8 r n}-\sigma_{r, n}\right\|_{L_{1}(I)} \geqslant r n 2^{-(s-1) s / 2-1}(8 r n)^{-s} 2^{-s+1}(8 r n)^{-1}=c n^{-s}
$$

где $c=r^{-s} 2^{-(s-1) s / 2-4 s-3}$.

Допустим теперь, что объединение всех компонент из группы $G_{+}^{\prime \prime}\left(\sigma_{r, n}\right)$ пересекается более чем с $2 r n$ интервалами $I_{i}$, на которых функция $\varphi_{s, 8 r n}$ является положительной. Тогда этому объединению принадлежит не менее чем $r n$ интервалов $I_{i}$, на которых функция $\varphi_{s, 8 r n}$ отрицательна. В этом случае из $(4.7)$ и $(4.8)$ будет следовать, что

$$
\left\|\varphi_{s, 8 r n}-\sigma_{r, n}\right\|_{L_{1}(I)} \geqslant r n 2^{-(s-1) s / 2-1}(8 r n)^{-s} 2^{-s+1}(8 r n)^{-1}=c n^{-s},
$$

где $c$ - та же константа, что и в (4.14).

Но тогда из (4.14) и (4.15) в силу произвольности выбора сплайна $\sigma_{r, n} \in \Sigma_{r, n}(I)$ следует оценка (4.13). Подставляя неравенство (4.13) в (4.12), имеем

$$
E\left(y_{s, 8 r n}, \Sigma_{r, n}\right)_{L_{1}(I)} \geqslant c n^{-s}, \quad n \geqslant 1, \quad r>s,
$$

где $c=c(r, s)$. А так как $y_{s, 8 r n} \in \Delta_{+}^{s} B_{\infty}(I)$, то очевидно, что

$$
E\left(\Delta_{+}^{s} B_{\infty}, \Sigma_{r, n}\right)_{L_{1}} \geqslant c n^{-s}, \quad n \geqslant 1, \quad r>s,
$$


где $c=c(r, s)$. В качестве простого следствия из этой оценки получаем оценку

$$
E\left(\Delta_{+}^{s} B_{p}, \Sigma_{r, n}\right)_{L_{q}} \geqslant c n^{-s}, \quad n \geqslant 1, \quad 1 \leqslant q<p \leqslant \infty, \quad r>s
$$

где $c=c(r, s, p, q)$. Объединяя оценки (4.6) и (4.16), имеем

$$
E\left(\Delta_{+}^{s} B_{p}, \Sigma_{r, n}\right)_{L_{q}} \geqslant c n^{-\min \{r, s\}}, \quad n \geqslant 1, \quad 1 \leqslant q<p \leqslant \infty, \quad r, s \in \mathbb{N},
$$

где $c=c(r, s, p, q)$. Таким образом, оценки снизу в теореме 1 доказаны полностью.

Соотношение (1.1) получаем, объединяя оценку сверху (3.61) с оценкой снизу (4.17). Теорема 1 доказана.

\section{§5. Доказательство оценок сверху в теореме 2}

Если $1 \leqslant n<s$, то для любой функции $x \in \Delta_{+}^{s} B_{p}(I), 1 \leqslant p \leqslant \infty$, заданной на интервале $I:=(-1,1)$, полагаем

$$
\rho_{n}(t ; x):=0, \quad t \in I
$$

Тогда из неравенства Гёльдера следует, что

$$
\left\|x(\cdot)-\rho_{n}(\cdot ; x)\right\|_{L_{q}(I)} \leqslant 2^{1 / q-1 / p}\|x\|_{L_{p}(I)}, \quad 1 \leqslant n<s, \quad 1 \leqslant q<p \leqslant \infty .
$$

Для доказательства оценок сверху при $n \geqslant s$ воспользуемся уже доказанньми вьше теоремой 1 и леммой 8 . Из теоремы 1 следует, что

$$
E\left(x, \Sigma_{s, k}\right)_{L_{q}(I)} \leqslant c k^{-s}, \quad k \geqslant 1, \quad 1 \leqslant q<p \leqslant \infty,
$$

где $c=c(s, p, q)$. Но тогда при $\lambda>s$ будут справедливы неравенства

$$
\sum_{k=1}^{n} k^{\lambda-1} E\left(x, \Sigma_{s, k}\right)_{L_{q}(I)} \leqslant c \sum_{k=1}^{n} k^{\lambda-1-s} \leqslant c_{*} n^{\lambda-s},
$$

где $c_{*}=c_{*}(s, \lambda, p, q)$. Поэтому из леммы 8 следует, что при любом фиксированном $\lambda>s$ имеет место оценка

$$
E\left(x, R_{n}\right)_{L_{q}(I)} \leqslant c^{*} n^{-s}, \quad n \geqslant s, \quad 1 \leqslant q<p \leqslant \infty,
$$

где $c^{*}=c^{*}(s, \lambda, p, q)$. Объединяя неравенства (5.1) и (5.2), имеем

$$
E\left(x, R_{n}\right)_{L_{q}(I)} \leqslant c n^{-s}, \quad n \geqslant 1, \quad 1 \leqslant q<p \leqslant \infty,
$$

где $c=c(s, \lambda, p, q)$. А так как $x-$ произвольная функция из $\Delta_{+}^{s} B_{p}(I)$, то

$$
E\left(\Delta_{+}^{s} B_{p}, R_{n}\right)_{L_{q}} \leqslant c n^{-s}, \quad n \geqslant 1, \quad 1 \leqslant q<p \leqslant \infty
$$

где $c=c(s, \lambda, p, q)$. Таким образом, необходимые оценки сверху в теореме 2 доказаны. 


\section{§6. Доказательство оценок снизу в теореме 2}

Оценки снизу доказываются аналогично тому, как это сделано для сплайнов $\sigma_{r, n} \in \Sigma_{r, n}(I)$ в случае $r>s$. Пусть $\varphi_{s, m}, y_{s}$ и $y_{s, m}$ - функции, определенные в $(4.8),(4.10)$ и (4.11) соответственно. Учитьвая, что $y_{s}$ - алгебраический многочлен степени $s$, имеем

$$
E\left(y_{s, m}, R_{n}\right)_{L_{1}(I)} \geqslant E\left(\varphi_{s, m}, R_{n+s+1}\right)_{L_{1}(I)}, \quad n, m, s \in \mathbb{N}
$$

Полагая $m=16(n+s+1)$ и учитывая, что любая рациональная функция $\rho_{n+s+1} \in$ $R_{n+s+1}$ знакопостоянна на не более чем $2(n+s+1)$ различных интервалах из $I$, приходим с помощью рассуждений из $\S 4$ к оценке

$$
E\left(\varphi_{s, 16(n+s+1)}, R_{n+s+1}\right)_{L_{1}(I)} \geqslant c(n+s+1)^{-s}, \quad n \geqslant 1
$$

где $c=c(s)$. Но тогда из (6.1) и (6.2) следует, что

$$
E\left(y_{s, 16(n+s+1)}, R_{n}\right)_{L_{1}(I)} \geqslant c n^{-s}, \quad n \geqslant 1,
$$

где $c=c(s)$. А так как $y_{s, 16(n+s+1)} \in \Delta_{+}^{s} B_{\infty}(I)$, то оценка

$$
E\left(\Delta_{+}^{s} B_{p}, R_{n}\right)_{L_{q}} \geqslant c n^{-s}, \quad n \geqslant 1, \quad 1 \leqslant q<p \leqslant \infty
$$

где $c=c(s, p, q)$, является простым следствием из (6.3). Итак, необходимая оценка снизу в теореме 2 доказана.

Соотношение (1.2) получаем, объединив оценки (5.3) и (6.4). Этим завершаем доказательство теоремы 2.

\section{Список литературы}

1. Bullen P. S. A criterion for $n$-convexity // Pacific J. Math. 1971. V. 36. P. 81-98.

2. Roberts A. W., Varberg D. E. Convex functions. New York: Academic Press, 1973.

3. Pečarić J. E., Proschan F., Tong Y. L. Convex functions, partial orderings, and statistical applications. Boston: Academic Press, 1992. (Math. Sci. Engrg. V. 187.)

4. Буланов А. П. О порядке приближения выпуклтх функций рациональыми функциями // Изв. АН СССР. Сер. матем. 1969. Т. 33. № 5. С. 1132-1148.

5. Попов В. А., Петрушев П. П. Точный порядок наилучшего равномерного приближения выпуклых функций рациональными функциями // Матем. сб. 1977. Т. 103(145). № 2 (6). C. $285-292$.

6. Ivanov K. G. Approximation of convex functions by means of polynomials and polygons // Approximation and function spaces. Proceedings of Conference (Gdansk, 1979). Warszawa, 1981. P. 287-293.

7. Hu Y. Convexity preserving approximation by free knot splines // SIAM J. Math. Anal. 1991. V. 22. P. $1183-1191$.

8. Konovalov V. N., Leviatan D. Kolmogorov and linear widths of weighted Sobolev-type classes on a finite interval. II // J. Approx. Theory. 2001. V. 113. P. 266-297.

9. Konovalov V. N., Leviatan D. Estimates on the approximation of 3-monotone functions by 3-monotone quadratic splines // East J. Approx. 2001. V. 7. P. 333-349.

10. Konovalov V.N., Leviatan D. Shape-preserving widths of weighted Sobolev-type classes of positive, monotone and convex functions on a finite interval // Constr. Approx. 2003. V. 19. P. $23-58$. 
11. Konovalov V. N., Leviatan D. Shape preserving widths of Sobolev-type classes of $s$-monotone functions on a finite interval // Israel J. Math. 2003. V. 133. P. 239-268.

12. Konovalov V. N., Leviatan D. Free-knot splines approximation of $s$-monotone functions on a finite interval // Adv. Comput. Math. 2004. V. 20. P. 347-366.

13. Konovalov $V . N$. Shape preserving widths of Kolmogorov-type of the classes of positive, monotone, and convex integrable functions // East J. Approx. 2004. V. 10. № 1-2. P. 93-117.

14. Коновалов В. Н. Формосохраняющие поперечники типа Колмогорова классов $s$-монотонных интегрируемых функций // Укр. матем. журн. 2004. Т. 55. № 7. С. 901-926.

15. Харди Г., Литтлвуд Д., Полиа Г. Неравенства. М.: ИЛ, 1948.

16. Пекарский $A$. A. Соотношения между наилучшими рациональньми и кусочно-полиномиальньми приближениями // Весці Акадэміi навук БССР. Сер. фіз.-матем. навук. 1986. № 5. C. $36-39$.

17. Petrushev P. P. Relation between rational and spline approximation in $L_{p}$ metric // J. Approx. Theory. 1987. V. 50. P. 141-159.

18. Тихомиров В. М. Некоторые вопросы теории приближений. М.: МГУ, 1976.

Институт математики НАН Украины, г. Киев

Поступила в редакцию

E-mail: vikono@imath.kiev.ua

10.06.2004 и 01.09.2004 